\title{
Free energy of a holonomous plasma
}

\author{
Chris P. Korthals Altes \\ Aix Marseille Univ, Université de Toulon, CNRS, CPT, Marseille, France \\ and NIKHEF theory group, P.O. Box 41882, 1009 DB Amsterdam, Netherlands
}

Hiromichi Nishimura $\odot$

Research and Education Center for Natural Sciences, Keio University, Toyko, Japan and RIKEN BNL Research Center, Brookhaven National Laboratory, Upton, New York 11973, USA

Robert D. Pisarski®

Department of Physics, Brookhaven National Laboratory, Upton, New York 11973, USA

Vladimir V. Skokov

Department of Physics, North Carolina State University, Raleigh, North Carolina 27695, USA and RIKEN BNL Research Center, Brookhaven National Laboratory, Upton, New York 11973, USA

(Received 11 February 2020; accepted 1 May 2020; published 27 May 2020)

\begin{abstract}
At a nonzero temperature $T$, a constant field $\bar{A}_{0} \sim T / g$ generates nontrivial eigenvalues of the thermal Wilson line. We discuss contributions to the free energy of such a holonomous plasma when the coupling constant, $g$, is weak. We review the computation to $\sim g^{2}$ by several alternate methods, and show that gauge invariant sources, which are nonlinear in the gauge potential $A_{0}$, generate novel contributions to the gluon self-energy at $\sim g^{2}$. These ensure the gluon self-energy remains transverse to $\sim g^{2}$, and are essential in computing contributions to the free energy at $\sim g^{3}$ for small holonomy, $\bar{A}_{0} \sim T$. We show that the contribution $\sim g^{3}$ from off-diagonal gluons is discontinuous as the holonomy vanishes. The contribution from diagonal gluons is continuous as the holonomy vanishes, but sharply constrains the possible sources which generate nonzero holonomy, and must involve an infinite number of Polyakov loops.
\end{abstract}

DOI: 10.1103/PhysRevD.101.094025

\section{INTRODUCTION}

The collisions of heavy nuclei at very high energies demonstrate the existence of a qualitatively new state of matter. It is most natural to assume that this is the production of a quark-gluon plasma (QGP) which is, at least approximately, in thermal equilibrium at a temperature $T$. The properties of the QGP can be computed perturbatively in the coupling constant $g$ [1-4], but this is only useful at very high temperature. At lower temperature, resummation is imperative [5,6], but this again fails at temperatures several times the transition temperature, which can be termed a "semi"-QGP. Numerical simulations on the lattice [7] provide detailed information at all temperatures in equilibrium, but at present this is much harder near equilibrium, such as to compute transport coefficients.

Published by the American Physical Society under the terms of the Creative Commons Attribution 4.0 International license. Further distribution of this work must maintain attribution to the author(s) and the published article's title, journal citation, and DOI. Funded by SCOAP .
In the pure gauge theory the order parameter for deconfinement are Polyakov loops. In an $S U(N)$ gauge theory, up to global $Z(N)$ rotations these are near unity at high temperature, and, if charged under $Z(N)$, vanish in the confined phase. Thus the semi-QGP is characterized by nonzero holonomy for Polyakov loops, where they are nonzero but less than unity.

To treat such a holonomous plasma, it is most natural to take a constant, background field for the vector potential, $\bar{A}_{0} \sim \Theta T / g$, where $\Theta$ is a diagonal, traceless color matrix [8-22]. In this paper we consider the analysis of a holonomous plasma in perturbation theory.

The computation of the holonomous potential at leading order is reviewed in Sec. II, mainly to establish notation $[8,9]$. It is atypical, as a potential for holonomy first arises then. The computation at $\sim g^{2}$ is given in Sec. III [10-25]. We use several different methods, and show that the potential is only gauge invariant in the presence of gauge invariant sources involving the Polyakov loops. Because these are nonlinear functions of the gauge field, these generate new contributions to the gluon self-energy $\sim g^{2}$. These are nonlocal, but essential 
in showing that the gluon self-energy remains transverse to this order.

If the holonomy is large, $\Theta \sim 1$, then the contribution of the off-diagonal gluons to the free energy is a power series in $g^{2}$. If the holonomy is weak, however, $\Theta \sim g$, then there are contributions to the free energy $\sim g^{3}$, as in the perturbative vacuum [2]. Previously we demonstrated that a novel result occurs at this order [24]: the contribution from offdiagonal gluons jumps discontinuously as the holonomy goes to zero. In Sec. IV we demonstrate this surprising result by another more direct means from that in Ref. [24], using hard thermal loops [26].

In Sec. IV B we show that while the contribution from diagonal gluons vanishes smoothly with the holonomy, that this requires rather nontrivial constraints on the associated sources. We are able to establish rigorous constraints for two $[27,28]$ and an infinite number of colors [29-31]. For the latter we use methods from matrix models $[32,33]$. The conclusion is that for the holonomy to turn on smoothly for a weak source, that the source must involve a sum over an infinite number of Polyakov loops.

Understanding the behavior of a holonomous plasma is of intrinsic interest in understanding the behavior of gauge theories at nonzero temperature. It is also of use in developing effective theories, which can then be analytically continued to compute properties near equilibrium [24,34-39]. These effective theories involve a perturbative potential for the holonomous potential, in addition to a nonperturbative term, added by hand, which drives the transition to confinement. Thus the present analysis will help in refining such effective theories. Notably, the source used as a nonperturbative holonomous potential in these models satisfies that required by the analysis of Sec. IV B.

While in this paper we do not consider dynamical quarks, their contribution to the holonomous potential can be computed directly, including at nonzero density $[22,23]$. Doing so, one finds that the effective theory developed for the pure gauge theory gives a reasonable analysis of QCD, with three flavor of light quarks [39].

An analysis with the insertion method is treated separately [25]. This allows one to show that the free energy from off-diagonal contributions is continuous to $\sim g^{4}$ as the holonomy vanishes.

\section{ONE LOOP ORDER}

To compute the effective potential one can either use an external source or a constrained path integral. Of course these must be equivalent, but this is not evident at two loop order and beyond.

\section{A. External source}

In the presence of an external source $J_{\mu}$, the Lagrangian for a gauge field is
$\mathcal{L}=\frac{1}{2} \operatorname{tr} G_{\mu \nu}^{2}+\operatorname{tr} J^{\mu} A_{\mu}, \quad G_{\mu \nu}=\partial_{\mu} A_{\nu}-\partial_{\nu} A_{\mu}-i g\left[A_{\mu}, A_{\nu}\right]$

We consider a $S U(N)$ gauge theory, with the generalization to other gauge groups direct.

At a nonzero temperature $T$ gauge invariant quantities are given by traces of powers of the thermal Wilson line, which are Polyakov loops:

$$
\begin{aligned}
& \ell_{r}(x)=\frac{1}{N} \operatorname{tr} \mathbf{L}^{r}(x) \\
& \mathbf{L}(x)=\operatorname{tr} \mathcal{P} \exp \left(i g \int_{0}^{1 / T} A_{0}(x, \tau) d \tau\right) .
\end{aligned}
$$

In a holonomous plasma we expand the gauge potential about a classical field, $\bar{A}_{0}$, and a quantum field, $\mathcal{A}_{\mu}$,

$$
A_{\mu}=\bar{A}_{\mu}+\mathcal{A}_{\mu}, \quad \bar{A}_{\mu}=\delta_{\mu 0} \Theta \frac{T}{g} .
$$

The classical field $\bar{A}_{0}$ is constant, with $\Theta$ a diagonal, traceless matrix: $\Theta^{a b}=\theta^{a} \delta^{a b}, \quad \sum_{a=1}^{N} \theta^{a}=0$. In this background,

$$
\overline{\mathbf{L}}^{r}=\mathrm{e}^{i r \Theta} .
$$

We use background field gauge, with the gauge dependent terms

$$
\mathcal{L}_{\text {gauge }}=\frac{1}{\xi} \operatorname{tr}\left(\bar{D}_{\mu} \mathcal{A}_{\mu}\right)^{2}+\bar{\eta}\left(-\bar{D}_{\mu} D_{\mu}\right) \eta
$$

where $D_{\mu}=\partial_{\mu}-i g\left[A_{\mu},\right]$ and $\bar{D}_{\mu}=\partial_{\mu}-i g\left[\bar{A}_{\mu},\right]$.

The generating functional $\mathcal{W}(J)$ is defined by

$$
\begin{aligned}
\exp (\mathcal{W}(J))= & \int \mathcal{D} A_{\mu} \mathcal{D} \eta \mathcal{D} \bar{\eta} \exp \\
& \times\left(-\int_{0}^{1 / T} d \tau \int d^{3} x\left(\mathcal{L}+\mathcal{L}_{\text {gauge }}\right)\right) .
\end{aligned}
$$

To one loop order the computation proceeds by integrating over the $A_{\mu}^{\text {qu }}$ to quadratic order. This is standard, and we only wish to make the following comments.

Assume that the background field is nontrivial, such as for an instanton. Then the associated field strength $G_{\mu \nu} \sim 1 / g$, and the equation of motion is

$$
\bar{D}_{\mu} \bar{G}_{\mu \nu}=J_{\nu} .
$$

For this to be consistent, $J_{\nu} \sim 1 / g$.

For a constant, diagonal $\bar{A}$ field, though, the classical field strength vanishes identically. We then assume that the source is not $\sim 1 / g$, but $\sim 1$. As we show later, the source 
does not contribute at leading order, but it does at next to leading order.

Integrating over $Q_{\mu}$, we obtain the effective action to one loop order

$$
\mathcal{S}_{\text {eff }}=-\operatorname{tr} \log \left(-\left(\bar{D}_{\mu}\right)^{2}\right) .
$$

This is the determinant from gluon and ghost fields. It can be shown rather directly that this expression is independent of the gauge fixing parameter, $\xi$.

Define

$$
\mathcal{W}(J)=\int d^{3} x\left(-V(A)+\operatorname{tr} J_{\mu} A_{\mu}\right)
$$

At one loop order, the holonomous potential is

$$
\mathcal{V}_{1}(\Theta)=-\frac{T^{3}}{\pi^{2}} \sum_{n=1}^{\infty} \frac{1}{n^{4}}\left|\operatorname{tr} \overline{\mathbf{L}}^{n}\right|^{2}=\frac{2 \pi^{2} T^{3}}{3} \sum_{a, b=1}^{N} B_{4}\left(\frac{\theta_{a}-\theta_{b}}{2 \pi}\right),
$$

where $B_{4}$ is the fourth Bernoulli polynomial,

$$
B_{4}(x)=-\frac{1}{30}+x^{2}(1-|x|)^{2} .
$$

In Eq. (10) $\theta_{a}-\theta_{b}$ is defined modulo $2 \pi$, since in the thermal Wilson line the $\theta_{a}$ are angular variables.

The one particle irreducible (1PI) generating functional is the Legendre transformation of $\mathcal{W}(J)$,

$$
\Gamma\left(\tilde{A}_{0}\right)=\sup \left(\int d^{3} x \operatorname{tr}\left(J_{\mu} \tilde{A}_{0}\right)-\mathcal{W}(J)\right) .
$$

Here sup denotes that one finds the point extremal with respect to variations in $J$. Usually the field is a function of $J$. Here because of the degeneracy at leading order, though, $\tilde{A}_{0}$ is independent of $J$. Hence the variation is trivial, and simply imposes $\tilde{A}_{0}=\bar{A}_{0}$, giving

$$
\Gamma\left(\tilde{A}_{0}\right)=\mathcal{V}_{1}(\Theta) .
$$

Note the distinction with the usual effective potential: we do not use the equations of motion to require that the variation of $\mathcal{V}_{1}\left(\bar{A}_{0}(J)\right)$ is extremal with respect to $J$.

Beyond leading order, the potential $\mathcal{V}_{1}(\Theta)$ lifts the degeneracy.

\section{B. Constrained functional integral}

Another way of computing is to constrain the value of the spatial average of the Polyakov loop. To avoid clutter we constrain only $\ell_{1}$, with the complete generalization given below, Eq. (20). The constrained functional integral is

$$
\begin{aligned}
\exp (-V \mathcal{V}(\bar{\ell}))= & \int \mathcal{D} A_{\mu} \mathcal{D} \eta \mathcal{D} \bar{\eta} \delta\left(\bar{\ell}-\int \frac{d^{3} x}{V} \ell_{1}(x)\right) \\
& \times \exp \left(-\int_{0}^{1 / T} d \tau \int d^{3} x\left(\mathcal{L}+\mathcal{L}_{\text {gauge }}\right)\right)
\end{aligned}
$$

We exponentiate the constraint,

$$
\begin{aligned}
\delta(\bar{\ell} & \left.-\int \frac{d^{3} x}{V} \ell_{1}(x)\right) \\
& =\int d \epsilon \exp \left(i \epsilon\left(\bar{\ell}-\int \frac{d^{3} x}{V} \ell_{1}(x)\right)\right) .
\end{aligned}
$$

Since we constrain only the spatial average of the loop(s), there is only a single constraint field, $\epsilon . V$ is the spatial volume.

We expand the constraint field,

$$
\epsilon=\epsilon^{\mathrm{cl}}+\epsilon^{\mathrm{qu}} .
$$

A nonzero value of $\epsilon^{\mathrm{cl}}$ acts like an external source. Since there is no potential for $\bar{A}$ at leading order, this source vanishes at leading order,

$$
\epsilon^{\mathrm{cl}}=0
$$

As before, it is direct to compute the constrained partition function. Integrating over $\epsilon^{\mathrm{qu}}$ imposes the constraint on the loop, requiring $\bar{A}$ to give the requisite value of the loop, $\ell_{1}$. Integration over $A_{\mu}^{\mathrm{qu}}$ is trivial, because the equation of motion vanishes anyway. The integration over $A_{\mu}^{\text {qu }}$ is also unaffected, as the constraint field does not contribute, $\epsilon^{\mathrm{cl}}=0$. The result is that of Eq. (13).

\section{TWO LOOP ORDER}

The computation of the free energy to $\sim g^{2}$ is an old story $[10-22,40]$. Nevertheless, as we show there are subtleties in the computation of the gluon self-energy to the same order. Thus we summarize the computation briefly in order to introduce behavior of the gluon self-energy to this order. This is essential in order to compute corrections to higher loop order, starting at $\sim g^{3}$.

\section{A. Linear gauge}

To two loop order, the result for the potential is

$$
\begin{aligned}
\mathcal{V}_{2}^{\text {pert }}(\Theta)= & \frac{g^{2} T^{3}}{4} \sum_{a, b, c=1}^{N} B_{2}\left(\frac{\theta_{a}-\theta_{c}}{2 \pi}\right) B_{2}\left(\frac{\theta_{b}-\theta_{c}}{2 \pi}\right) \\
& +(1-\xi) B_{1}\left(\frac{\theta_{a}-\theta_{c}}{2 \pi}\right) B_{3}\left(\frac{\theta_{b}-\theta_{c}}{2 \pi}\right)
\end{aligned}
$$


This involves the first, second, and third Bernoulli polynomials,

$$
\begin{aligned}
& B_{1}(x)=-\frac{1}{2} \operatorname{sign}(x)+x \\
& B_{2}(x)=\frac{1}{6}-|x|+x^{2} \\
& B_{3}(x)=\frac{1}{2} x-\frac{3}{2} \operatorname{sign}(x) x^{2}+x^{3} .
\end{aligned}
$$

Each difference of the $\theta$ 's, such as $\theta_{a}-\theta_{b}$, is defined modulo $2 \pi$. Even Bernoulli polynomials are even in $x$, and so depend only upon $\left|\theta_{a}-\theta_{b}\right|$. Odd Bernoulli polynomials are odd in $x$.

The potential in Eq. (18) is rather unexpected since it explicitly depends on $\xi$. It can also be shown that there is a minimum at a nonzero value of $q \sim(3-\xi) g^{2}$. Note however that the pressure is $\xi$ independent to order $g^{2}$.

The $\xi$ dependence can be understood from the Nielsen identities $[15,16,41]$. For a value of $\theta \sim g^{2}$, it contributes to the potential at $\sim g^{4}$. Nevertheless, it is useful to see how this a gauge invariant result arises explicitly. Doing so we show that the usual perturbative vacuum is stable.

\section{B. Constrained functional integral}

Since the above source and potential are gauge variant, we introduce gauge invariant constraints into the action. For $S U(N)$ we constrain the Polyakov loops by adding constraint fields $\epsilon_{r}$ to the action,

$$
\mathcal{S}_{\text {cons }}=i \sum_{r=1}^{N} \epsilon_{r}\left(\ell_{r}-\int \frac{d^{3} x}{V} \operatorname{tr} \mathbf{L}^{r}(x)\right) .
$$

Only $N-1$ constraints are needed, but we find it convenient to use one too many constraints, from $r=1$ to $N$ instead of $N-1$. This is done for the following reason. For $S U(N)$ the sum of the $\theta_{a}$ 's vanishes, and there are only $N-1$ independent $\theta_{a}$ 's. It is awkward to eliminate one of the $N \theta_{a}$ 's in favor of the independent variables, though. Instead, it is easier to pretend as if all of the $N \theta_{a}$ are independent, and derive the equations of motion for the $N \theta_{a}$.

\section{Insertion method}

The insertion method is a straightforward expansion of the gauge action and the constraint in terms of the fluctuation fields $\mathcal{A}_{\mu}$ and $\epsilon^{\mathrm{qu}} \quad[17-22,25]$. This gives constant terms, linear terms, quadratic terms, and interaction terms. The linear terms are set to zero, and fixes $\epsilon^{\mathrm{cl}}=0$, as in Eq. (17), and $\bar{A}$ in terms of $\ell$. The quadratic terms in the action now include $\mathcal{L}_{\text {quadr }}-i \epsilon^{\text {qu }} \mathcal{A}_{0}(0)$, where $\mathcal{A}_{0}(0)$ is the zero momentum component of the fluctuation field. Vertices are generated by expanding the gauge action plus the constraint,

$$
\mathcal{L}_{\text {int }}+i \epsilon^{\mathrm{qu}}\left(\mathbf{L}_{2}+\mathbf{L}_{3}+\cdots\right)
$$

where the subscripts indicate the powers of the quantum fluctuation $\mathcal{A}_{\mu}$. Then the integration over $\epsilon^{\mathrm{qu}}$ is done. This reinstates the delta function of the original constraint but now in the simple form $\delta\left(\mathcal{A}_{0}(0)\right)$ times the pure gauge field vertices. It also introduces also new vertices, where $\epsilon^{\mathrm{qu}}$ multiplies $\mathcal{L}_{\text {int }}\left(\mathbf{L}_{2}+\cdots\right)$; this generates derivatives of the delta-function. The derivatives in $\mathcal{A}_{0}(0)$ act through integration by parts on the gauge interaction vertices and on the Polyakov loops. These are called the insertion vertices: integration over the remaining fluctuations gives then, apart from the usual QCD diagrams, "insertion diagrams" [17-22,25]. These are key to understanding how gauge invariance is implemented. The insertion terms do generate contribution to the two, three, and higher point functions of the gluons. Up to and including three loop order the thermodynamic limit poses no problems, except in the case of diagonal gluons with two self-energy insertions. There, the finite size corrections to the self-energy have to be taken into account and guarantee a well-defined $\mathcal{O}\left(g^{3}\right)$ contribution [25].

\section{Alternate approach}

In the insertion approach, $\epsilon^{\mathrm{cl}}=0$, Eq. (17), order by order in perturbation theory. An alternate approach is the following. Since the degeneracy in $\Theta$ is broken at one loop order, we generalize Eq. (10) from a function of the background field, $\bar{A}_{0}$, to a function of the full vector potential, $A_{\mu}=\bar{A}_{\mu}+\mathcal{A}_{\mu}$ :

$$
\mathcal{V}_{1}\left(A_{0}\right)=-\frac{T^{3}}{\pi^{2}} \int d^{3} x \sum_{n=1}^{\infty} \frac{1}{n^{4}}\left|\operatorname{tr} \mathbf{L}^{n}(x)\right|^{2} .
$$

We then add and subtract $\mathcal{V}_{1}\left(A_{0}\right)$ to the Lagrangian. The subtracted term cancels $\mathcal{V}_{1}\left(\bar{A}_{0}\right)$ the same term when it is generated at one loop order. This is exactly analogous to how, for example, a Debye mass is included in perturbation theory.

The advantage of adding $\mathcal{V}_{1}\left(A_{0}\right)$ is that the degeneracy with respect to $\bar{A}_{0}$, valid at the classical level, is lifted. The equations of motion are now

$$
-\frac{i}{V} \sum_{r=1}^{N} i \epsilon_{r}^{\mathrm{cl}} r \mathrm{e}^{i r \theta_{a}}=\frac{8 \pi T^{3}}{3} \sum_{b=1}^{N} B_{3}\left(\frac{\theta_{a}-\theta_{b}}{2 \pi}\right) ;
$$

$B_{3}$ is the third Bernoulli polynomial, which arises as the derivative of $B_{4}(x)$. There are $N$ equations of motion in Eq. (23). As an odd Bernoulli polynomial, $B_{3}(x)$ is defined to be odd in $x$, and so by summing over $a$, we obtain

$$
\sum_{r=1}^{N} \sum_{a=1}^{N} r \epsilon_{r}^{\mathrm{cl}} \mathrm{e}^{i r \theta_{a}}=0
$$


In principle it is possible to eliminate one of the $\theta_{a}$ 's for the $N-1$ independent variables. As we shall see, however, unexpectedly there is no need to explicitly do so, nor to solve for the values of the constraint fields $\epsilon_{r}$. This greatly simplifies matters.

The equation of motion in Eq. (23) is identical to that with an external source $\mathcal{J}_{r}$ which couples to the Polyakov loop $\ell_{r}$, with $\epsilon_{r}^{\mathrm{cl}}=i \mathcal{J}_{r} V$. This was the approach used in our previous work [24]. With a constraint action, it is natural that the expectation value of the classical field is imaginary and proportional to the spatial volume. Also notice that the source $\mathcal{J}_{r}$ are naturally of order one, and not $\sim 1 / g$, in agreement with the analysis in Sec. II A. With either a constraint or a source, however, it is necessary to explicitly add the one loop term to lift the degeneracy in $\Theta$. Thus adding Eq. (22) above is equivalent to Eq. (18) of Ref. [24].

\section{Expansion of Polyakov loops to quadratic order}

The major difference between a source that couples to Polyakov loops, and the usual term which is linear in $A_{\mu}$, is that Polyakov loops are gauge invariant and hence a nonlocal, infinite power series in $\mathcal{A}_{0}$. To $\sim g^{2}$, it is necessary to include terms of quadratic order in $\mathcal{A}_{0}$, and so on to higher order. In this subsection we compute the terms to quadratic order.

We need the thermal Wilson for a time of limited extent, $\tau^{\prime}: 0 \rightarrow \tau$,

$$
\mathbf{L}(x, \tau)=\mathcal{P} \exp \left(i g \int_{0}^{\tau} A_{0}\left(x, \tau^{\prime}\right) d \tau^{\prime}\right) .
$$

where $\mathcal{P}$ denotes path ordering. For the $r$ th power of the Wilson line,

$$
\operatorname{tr} \mathbf{L}^{r}(x, 1 / T)=\operatorname{tr} \mathbf{L}(x, r / T) .
$$

We define the expansion about the classical field as

$$
\mathbf{L}^{r}(x, 1 / T)=\overline{\mathbf{L}}^{r}+\delta \mathbf{L}_{1}^{r}(x)+\delta \mathbf{L}_{2}^{r}(x)+\cdots ; \quad \overline{\mathbf{L}}^{r}=\mathrm{e}^{i r \Theta},
$$

where the subscript denotes the power of $\mathcal{A}_{0}$.

To linear order,

$$
\delta \mathbf{L}_{1}^{r}(x)=i g \int_{0}^{r / T} d \tau \overline{\mathbf{L}}(r / T-\tau) \mathcal{A}_{0}(x, \tau) \overline{\mathbf{L}}(\tau) .
$$

Taking the trace,

$$
\operatorname{tr} \delta \mathbf{L}_{1}^{r}(x)=i g r \operatorname{tr}\left(\mathrm{e}^{i r \Theta} \int_{0}^{1 / T} d \tau \mathcal{A}_{0}(x, \tau)\right) .
$$

As $\overline{\mathbf{L}}$ is diagonal, only diagonal elements of $\mathcal{A}_{0}$ contribute. The integral over $\tau$ projects out the constant mode in $\tau$ for
$\mathcal{A}_{0}(x, \tau)$. To derive the equations of motion, it is useful to shift $\theta_{a} \rightarrow \theta_{a}+\delta \theta_{a}(x)$, so that

$$
\operatorname{tr} \delta \mathbf{L}_{1}^{r}(x)=\sum_{a=1}^{N} i r \mathrm{e}^{i r \theta_{a}} \delta \theta_{a}(x),
$$

which gives the left-hand side of Eq. (23).

To proceed further we need to choose an explicit basis. We adopt the double line notation familiar at large $N$ to finite $N$. In the fundamental representation,

$$
\left(t^{a b}\right)_{c d}=\frac{1}{\sqrt{2}}\left(\delta^{a c} \delta^{b d}-\frac{1}{N} \delta^{a b} \delta^{c d}\right)
$$

$a, b, c, d \ldots=1 \ldots N$. An adjoint matrix is denoted by the pair of upper indices, $a b$. Hence there is one too many generators, $N^{2}$ in all instead of $N^{2}-1$. The normalization of off-diagonal generators is standard,

$$
\operatorname{tr}\left(t^{a b} t^{b a}\right)=\frac{1}{2}, \quad a \neq b .
$$

Because the double lines of $S U(N)$ are ordered in opposite directions, the indices flip when two generators are contracted.

This basis is overcomplete by one diagonal generator. Consequently the normalization of the diagonal generators is unusual, Eqs. (16) and (17) of Ref. [26]. However, it is easy just multiplying diagonal matrices together, and so at least to the order at which we work, this can be ignored.

For example, to quadratic order the diagonal elements are

$$
\begin{aligned}
\left(\delta \mathbf{L}_{2}^{r}\right)_{\text {diag }}= & -g^{2} \int_{0}^{r / T} d \tau_{1} \\
& \times \int_{0}^{\tau_{1}} d \tau_{2} \mathrm{e}^{i r \theta_{a}} \mathcal{A}_{0}^{a a}\left(x, \tau_{1}\right) \mathcal{A}_{0}{ }^{a a}\left(x, \tau_{2}\right) .
\end{aligned}
$$

For the modes constant in time path ordering does not matter, and this is elementary. Path ordering does enter for time dependent modes.

More interesting are the off-diagonal elements:

$$
\begin{aligned}
\left(\delta \mathbf{L}_{2}^{r}\right)_{\text {off }}= & -g^{2} \int_{0}^{r / T} d \tau_{1} \int_{0}^{\tau_{1}} d \tau_{2} \overline{\mathbf{L}}\left(r / T-\tau_{1}\right) \\
& \times \mathcal{A}_{0}\left(x, \tau_{1}\right) \overline{\mathbf{L}}\left(\tau_{1}-\tau_{2}\right) \mathcal{A}_{0}\left(x, \tau_{2}\right) \overline{\mathbf{L}}\left(\tau_{2}\right) .
\end{aligned}
$$

For each of the $\mathcal{A}_{0}$ 's we go from the imaginary time $\tau$ to momentum space,

$$
\begin{aligned}
& \mathcal{A}_{0}\left(x, \tau_{1}\right)=T \sum_{n=-\infty}^{+\infty} \mathrm{e}^{-i p_{0} \tau_{1}} \mathcal{A}_{0}\left(x, p_{0}\right), \quad p_{0}=2 \pi n T \\
& \mathcal{A}_{0}\left(x, \tau_{2}\right)=T \sum_{n^{\prime}=-\infty}^{+\infty} \mathrm{e}^{-i p_{0}^{\prime} \tau_{2}} \mathcal{A}_{0}\left(x, p_{0}^{\prime}\right), \quad p_{0}^{\prime}=2 \pi n^{\prime} T .
\end{aligned}
$$


Because the Wilson line is nonlocal in time, it is possible that terms where $p_{0} \neq p_{0}^{\prime}$ contribute. In contrast, since the terms are local in space, the spatial momenta of the two $\mathcal{A}_{0}$ 's are equal and opposite.

The color structure enters in two ways:

$$
\mathcal{A}_{0}^{b a}\left(p_{0}\right) t^{a b} \mathcal{A}_{0}^{a b}\left(p_{0}^{\prime}\right) t^{b a} ; \quad \mathcal{A}_{0}^{a b}\left(p_{0}\right) t^{b a} \mathcal{A}_{0}^{b a}\left(p_{0}^{\prime}\right) t^{a b} .
$$

There is no summation over repeated indices, as the color indices $a$ and $b$, with $a \neq b$, are fixed.

Begin with the first permutation. Since $\overline{\mathbf{L}}$ is a diagonal matrix,

$$
\overline{\mathbf{L}}(\tau) t^{a b}=\mathrm{e}^{i \theta_{a} \tau T} t^{a b} ; \quad t^{a b} \overline{\mathbf{L}}(\tau)=t^{a b} \mathrm{e}^{i \theta_{b} \tau T} .
$$

Thus the first permutation in Eq. (36) gives

$$
\begin{aligned}
- & g^{2} T^{2} \mathrm{e}^{i r \theta_{a}} \sum_{n, n^{\prime}=-\infty}^{+\infty} \int_{0}^{r / T} d \tau_{1} \mathrm{e}^{-i p_{0}^{a b} \tau_{1}} \\
& \times \int_{0}^{\tau_{1}} d \tau_{2} \mathrm{e}^{-i p_{0}^{\prime b a} \tau_{2}}\left(\mathcal{A}_{0}^{b a}\left(p_{0}\right) t^{a b} \mathcal{A}_{0}^{a b}\left(p_{0}^{\prime}\right) t^{b a}\right),
\end{aligned}
$$

where

$$
p_{0}^{a b}=T\left(2 \pi n+\theta_{a}-\theta_{b}\right), \quad p_{0}^{\prime b a}=T\left(2 \pi n^{\prime}+\theta_{b}-\theta_{a}\right) .
$$

The integral over $\tau_{2}$ is

$$
\int_{0}^{\tau_{1}} d \tau_{2} \mathrm{e}^{-i p_{0}^{\prime b a} \tau_{2}}=\frac{1}{-i p_{0}^{\prime b a}}\left(\mathrm{e}^{-i p_{0}^{\prime b a} \tau_{1}}-1\right) .
$$

Integrating over $\tau_{1}$,

$$
-g^{2} T^{2} \sum_{n, n^{\prime}=-\infty}^{+\infty}\left(r \mathrm{e}^{2 \pi i r q_{a}} \frac{1}{-i p_{0}^{\prime b a}} \delta\left(p_{0}+p_{0}^{\prime}\right)+\frac{1}{p_{0}^{a b} p_{0}^{\prime b a}}\left(\mathrm{e}^{i r \theta^{b}}-\mathrm{e}^{i r \theta^{a}}\right)\right)\left(\mathcal{A}_{0}^{b a}\left(p_{0}\right) t^{a b} \mathcal{A}_{0}^{a b}\left(p_{0}^{\prime}\right) t^{b a}\right) .
$$

The other ordering in Eq. (36) gives

$$
-g^{2} T^{2} \sum_{n, n^{\prime}=-\infty}^{+\infty}\left(r \mathrm{e}^{i r \theta_{b}} \frac{1}{i p_{0}^{a b}} \delta\left(p_{0}+p_{0}^{\prime}\right)+\frac{1}{p_{0}^{a b} p_{0}^{\prime b a}}\left(\mathrm{e}^{i r \theta^{a}}-\mathrm{e}^{i r \theta^{b}}\right)\right)\left(\mathcal{A}_{0}^{a b}\left(p_{0}^{\prime}\right) t^{b a} \mathcal{A}_{0}^{b a}\left(p_{0}\right) t^{a b}\right),
$$

where we relabel $p_{0} \leftrightarrow p_{0}^{\prime}$ and $a \leftrightarrow b$. This agrees with previous results, such as Eq. (3.12) of [17].

We now add the two orderings. With the normalization of Eq. (32), we find for the sum of off-diagonal elements

$$
\operatorname{tr}\left(\delta \mathbf{L}_{2}^{r}(x)\right)_{\text {off }}=-\frac{g^{2}}{4 i} \sum_{a \neq b=1}^{N} r\left(\mathrm{e}^{i r \theta_{a}}-\mathrm{e}^{i r \theta_{b}}\right) T \sum_{n=-\infty}^{+\infty} \frac{1}{p_{0}^{a b}}\left(\mathcal{A}_{0}^{b a}\left(x, p_{0}\right) \mathcal{A}_{0}^{a b}\left(x,-p_{0}\right)\right) .
$$

The second terms in Eqs. (41) and (42) are truly nonlocal in time, as $p_{0}+p_{0}^{\prime} \neq 0$ contribute. After taking the trace, however, these terms cancel: because of the energy denominator, the result is diagonal in $p_{0}$ and nonlocal in the Euclidean time.

This term is special to the off-diagonal modes. For example, for the diagonal modes which are constant in time, $p_{0}=p_{0}^{\prime}=0$, Eq. (33) reduces to

$$
-g^{2} \mathrm{e}^{i r \theta_{a}} r^{2}\left(\mathcal{A}_{0}^{a a}\right)^{2} \text {. }
$$

\section{Corrections to Polyakov loops and the free energy at $\sim g^{2}$}

The result in Eq. (43) is useful in several ways. We first show how the results above can be used to compute the free energy at $\sim g^{2}$ in two different, but equivalent ways. This is necessary to compute corrections at higher order, to $\sim g^{3}$.

Consider the constraint action of Eq. (20). As in Eq. (16), we expand the $N$ constraint fields in classical and quantum components,

$$
\epsilon_{r}=\epsilon_{r}^{\mathrm{cl}}+\epsilon_{r}^{\mathrm{qu}} .
$$

For each of the $r$ constraint fields, the classical value of $\epsilon_{r}^{\mathrm{cl}}$ is determined by varying with respect to $\mathcal{A}_{0}$, and is given by Eq. (23).

The terms linear in $\epsilon_{r}^{\mathrm{qu}}$ are

$$
\begin{aligned}
& i \sum_{r=1}^{N} \epsilon_{r}^{\mathrm{qu}}\left(\ell_{r}-\int \frac{d^{3} x}{V} \operatorname{tr}\left(\overline{\mathbf{L}}^{r}+\delta \mathbf{L}_{2}^{r}(x)+\cdots\right)\right) \\
& -i \sum_{r=1}^{N} \epsilon_{r}^{\mathrm{qu}} \int \frac{d^{3} x}{V} \operatorname{tr} \delta \mathbf{L}_{1}^{r}(x) .
\end{aligned}
$$

Consider the last term, which is quadratic in the quantum fields, $\sim \epsilon_{r}^{\mathrm{qu}} \mathcal{A}_{0}$. From the form of $\delta \mathbf{L}_{1}^{r}$ in Eq. (29), only the static, $p_{0}=0$ component of $\mathcal{A}_{0}$ enters. Further, the constraint is over the spatial average of $\delta \mathbf{L}_{1}^{r}$, which further projects out the zero momentum component of the spatial momentum, $\boldsymbol{p}=0$. Unlike the static component in $p_{0}$, which is of finite measure, the zero momentum component 
in $\boldsymbol{p}$ is of zero measure. Thus we can ignore this part of the integral over $\epsilon_{r}^{\mathrm{qu}}$.

To evaluate Eq. (46) we use Eq. (43) to find

$$
\left\langle\operatorname{tr}\left(\delta \mathbf{L}_{2}^{r}(x)\right)\right\rangle_{\text {off }}=(3-\xi) \frac{g^{2}}{8 \pi} \sum_{a \neq b=1}^{N} \operatorname{ire}^{i r \theta_{a}} B_{1}\left(\frac{\theta_{a}-\theta_{b}}{2 \pi}\right) .
$$

This first term in Eq. (46) has a simple physical interpretation: as discussed by Belyaev [12], it represents a correction to the constraint at $\sim g^{2}$. This can be implemented by going from a "bare" $\theta_{a}$ to a renormalized $\theta_{a}$. This shift is finite, but field and $\xi$ dependent. Using this shifted $\theta_{a}$ in the free energy obtained perturbatively, one obtains the result below, Eq. (50).

The shift in the $\theta_{a}$ 's is natural. While the thermal Wilson line is gauge dependent, the eigenvalues of the Wilson line are gauge invariant. Shifting the eigenvalues is one way of implementing this.

The same result is obtained by using the insertion method of Sec. III B 1. There instead of a shift in the eigenvalues, there are new diagrams from expanding the constraint $[17-22,25]$.

Lastly, there is a third method of computing the free energy to $\sim g^{2}$. With the method of Sec. III B 2, to $\sim 1$ the constraint field develops an expectation value, $\epsilon_{r}^{\mathrm{cl}} \neq 0$. Using Eq. (23), the quadratic term in Eq. (43) contributes to the action as

$$
\begin{gathered}
-\frac{4 \pi}{3} g^{2} T^{3} \sum_{a, b, c=1}^{N}\left(B_{3}\left(\frac{\theta_{a}-\theta_{c}}{2 \pi}\right)-B_{3}\left(\frac{\theta_{b}-\theta_{c}}{2 \pi}\right)\right) T \\
\times \sum_{n=-\infty}^{+\infty} \frac{1}{p_{0}^{a b}} \int d^{3} x\left(\mathcal{A}_{0}^{b a}\left(x, p_{0}\right) \mathcal{A}_{0}^{a b}\left(x,-p_{0}\right)\right) .
\end{gathered}
$$

Notice that the factor of $1 / V$ in the constraint is compensated by $\epsilon_{r}^{\mathrm{cl}} \sim V$ in Eq. (23). Rather surprisingly, this contribution is completely independent of the detailed form of the $\epsilon_{r}^{\mathrm{cl}}$ : once the equations of motion are imposed, they completely drop out. We generalize this later to sources involving two traces in Sec. III F.

By contracting two $\mathcal{A}_{0}$ fields together, Eq. (48) contributes to the holonomous potential. The result is gauge variant, and proportional to $\xi$,

$$
\mathcal{V}_{2}^{\text {cons }}(\Theta)=-(3-\xi) \frac{g^{2} T^{3}}{3} \sum_{a, b, c=1}^{N} B_{1}\left(\frac{\theta_{a}-\theta_{c}}{2 \pi}\right) B_{3}\left(\frac{\theta_{b}-\theta_{c}}{2 \pi}\right)
$$

After some juggling [20,21] of Bernoulli polynomials,

$$
\begin{aligned}
\mathcal{V}_{2}(\Theta) & =\mathcal{V}_{2}^{\text {pert }}(\Theta)+\mathcal{V}_{2}^{\text {cons }}(\Theta) \\
& =-\frac{5}{24} g^{2} T^{3} \sum_{a, b=1}^{N} B_{4}\left(\frac{\theta_{a}-\theta_{b}}{2 \pi}\right) .
\end{aligned}
$$

This is both independent of the gauge fixing parameter, $\xi$, and proportional to the potential at one loop order. As such, the perturbative vacuum $\Theta=0$ is stable.

Each $B_{n}$ can be written as a sum of double traces of the Wilson line. Thus the terms $\sim B_{2} B_{2}$ and $\sim B_{1} B_{3}$ involve four traces. The final form $\sim B_{4}$, though, only involves two traces. This has interesting implications for the solutions of the theory at infinite $N$, where potentials with only double traces are often soluble, at least in certain limits.

\section{E. Holonomous gluon self-energy at one loop order}

$$
\text { 1. } \xi=1
$$

The result of Eq. (48) is a contribution to the gluon selfenergy for $a \neq b$,

$$
\begin{aligned}
\Pi_{\mathrm{cons} ; 00}^{a b, c d}\left(p^{a b}\right)= & -\delta^{a d} \delta^{b c} \frac{1}{p_{0}^{a b}} \frac{4 \pi}{3} g^{2} T^{3} \sum_{e=1}^{N}\left(B_{3}\left(\frac{\theta_{a}-\theta_{e}}{2 \pi}\right)\right. \\
& \left.+B_{3}\left(\frac{\theta_{e}-\theta_{b}}{2 \pi}\right)\right)
\end{aligned}
$$

$p_{\mu}^{a b}=\left(p_{0}^{a b}, \boldsymbol{p}\right)$, Eq. (39). This term is constant in the spatial momentum $\boldsymbol{p}$, and so a $\delta$-function in space. With a constrained functional integral, this term only arises in recognizing that $\epsilon^{\mathrm{cl}} \neq 0$; with a source, that the value of the source must be included. They arise in the insertion method just by doing only the Wick contractions that produce a volume term [17,20-22,25]. Then only gluons radiated from the Polyakov loop stay uncontracted, as in Eq. (41) that leads to Eq. (51).

The part of the gluon self-energy from the constraint satisfies

$$
p_{0}^{a b} \Pi_{\mathrm{cons} ; 00}^{a b, c d}\left(p^{a b}\right)=-\delta^{a d} \delta^{b c} \frac{4 \pi}{3} g^{2} T^{3} \sum_{a, b, c=1}^{N}\left(B_{3}\left(\frac{\theta_{a}-\theta_{c}}{2 \pi}\right)+B_{3}\left(\frac{\theta_{c}-\theta_{b}}{2 \pi}\right)\right) .
$$

There is also the usual perturbative contribution. When $\xi=1$, this equals

$$
p_{\mu}^{a b} \Pi_{\mathrm{pert}, \xi=1 ; \mu \nu}^{a b, c d}\left(p^{a b}\right)=+\delta^{\nu 0} \delta^{a d} \delta^{b c} \frac{4 \pi}{3} g^{2} T^{3} \sum_{a, b, c=1}^{N}\left(B_{3}\left(\frac{\theta_{a}-\theta_{c}}{2 \pi}\right)+B_{3}\left(\frac{\theta_{c}-\theta_{b}}{2 \pi}\right)\right) .
$$


Clearly the contributions of Eqs. (52) and (53) cancel identically, so that the sum is transverse,

$$
\begin{aligned}
p_{\mu}^{a b} \prod_{\text {total }, \xi=1 ; \mu \nu}^{a b, c d}\left(p^{a b}\right)= & p_{\mu}^{a b}\left(\Pi_{\text {pert }, \xi=1 ; \mu \nu}^{a b, d}\left(p^{a b}\right)\right. \\
& \left.+\prod_{\text {cons } ; \mu \nu}^{a b, c d}\left(p^{a b}\right)\right)=0,
\end{aligned}
$$

where $\Pi_{\mathrm{cons} ; \mu \nu}=\delta^{\mu 0} \delta^{\nu 0} \Pi_{\mathrm{cons} ; 00}$.

\section{2. $\xi \neq 1$}

When $\xi \neq 1$, the analysis is more involved, but Eq. (54) remains valid in the limit of zero momentum. We begin with the perturbative vacuum, $\theta_{a}=0$. From the analysis of Elze, Heinz, Kajantie, and Toimela [10], in covariant gauges with $\xi \neq 1$, at nonzero temperature there is an additional contribution to the gluon self-energy. The contraction of $p_{\mu}$ with the gluon self-energy is proportional to

$$
\begin{gathered}
p_{\mu} \Pi_{\mathrm{pert}, \xi, \mu \nu}(p) \sim(1-\xi) g^{2} N \bigvee_{k} \frac{k \cdot r}{\left(k^{2}\right)^{2} r^{2}} k_{\mu} T_{\mu \nu}(p) ; \\
k_{\mu}+p_{\mu}+r_{\mu}=0 .
\end{gathered}
$$

Here we introduce the transverse tensor,

$$
T_{\mu \nu}(p)=\delta_{\mu \nu} p^{2}-p_{\mu} p_{\nu},
$$

and a shorthand for the loop integral at $T \neq 0$,

$$
y_{k}=T \sum_{n=-\infty}^{+\infty} \int \frac{d^{3} \boldsymbol{k}}{(2 \pi)^{3}}
$$

where $k^{\mu}=\left(k_{0}, \boldsymbol{k}\right), k_{0}=2 \pi n T$.

It can be shown that $p_{\mu} \Pi_{\text {pert }, \xi \mu \nu}(p)=0$ in the static limit, $p_{0}=0$ [10]. To do so, it is convenient to use

$$
2 k \cdot r=p^{2}-k^{2}-r^{2}
$$

to replace $k \cdot r$ in the numerator of Eq. (55). There are three terms which result. The second term, $\sim k^{2}$, gives a contribution of the type similar to that for $\xi=1$,

$$
\sim \bigcup_{k} \frac{1}{k^{2} r^{2}} k^{\mu} T_{\mu \nu}(p)
$$

The integral over $k$ gives a result $\sim p_{\mu}$, which vanishes when contracted with the transverse tensor $T_{\mu \nu}(p)$.

The third term in Eq. (58) is $\sim r^{2}$,

$$
\sim \bigcup_{k} \frac{1}{\left(k^{2}\right)^{2}} k_{\mu} T_{\mu \nu}(p)
$$

which vanishes because it is odd in $k_{\mu}$.
This leaves the first term in Eq. (58), $\sim p^{2}$.

$$
\sim p^{2} \bigvee_{k} \frac{1}{\left(k^{2}\right)^{2} r^{2}} k_{\mu} T_{\mu \nu}(p) \text {. }
$$

We consider the static limit, $p_{0}=0$. Then when $\mu=0$ the sum is odd in $k_{0}$ and so vanishes. When $\mu=i$ is a spatial index the integral $\sim p_{i}$, so for $\nu=0, p_{i} T_{i 0} \sim p_{i}^{2} p_{0}=0$; for $\nu=j, p_{i} T_{i j}(p)=p_{0}^{2} p_{j}$. In either case, the result vanishes at $p_{0}=0$.

This result generalizes to a nontrival background, $\theta_{a} \neq 0$. The momenta then depend upon the color, with the external momentum $p^{a b}=\left(p_{0}^{a b}, \boldsymbol{p}\right)$, with $p_{0}^{a b}=$ $\left(T\left(2 \pi n+\theta^{a}-\theta^{b}\right), \boldsymbol{p}\right)$, Eq. (39). We choose the loop momenta as $k^{b c}=\left(k_{0}^{b c}, \boldsymbol{k}\right)$, and define $r_{\mu}^{c a}$ through

$$
p_{\mu}^{a b}+k_{\mu}^{b c}+r_{\mu}^{c a}=0 .
$$

The conservation of the color dependent momenta follows trivially from the conservation of the usual momenta and the definition of $p^{a b}$, etc., Eq. (55) becomes

$$
\begin{aligned}
p_{\mu}^{a b} \Pi_{\mathrm{pert}, \xi, \mu \nu}\left(p^{a b}\right) \sim & (1-\xi) g^{2} \sum_{c} f^{(a b, b c, c a)} f^{(a c, c b, b a)} \\
& \times \bigcup_{k} \frac{k^{b c} \cdot r^{c a}}{\left(\left(k^{b c}\right)^{2}\right)^{2}\left(r^{c a}\right)^{2}} k_{\mu}^{b c} T_{\mu \nu}\left(p^{a b}\right) .
\end{aligned}
$$

The color sum involves the structure constants in the double line basis, the $f^{(a b, b c, c a)}$ [26], but their explicit form is inconsequential. The tensor $T_{\mu \nu}\left(p^{a b}\right)$ remains transverse in $p_{\mu}^{a b}$.

The proof proceeds as before, using

$$
2 k^{b c} \cdot r^{c a}=\left(p^{a b}\right)^{2}-\left(k^{b c}\right)^{2}-\left(r^{c a}\right)^{2}
$$

to simplify the numerator in the integral. The second term, $\sim\left(k^{b c}\right)^{2}$, becomes

$$
\sim \oint_{k} \frac{1}{\left(k^{b c}\right)^{2}\left(r^{c a}\right)^{2}} k_{\mu}^{b c} T_{\mu \nu}\left(p^{a b}\right)
$$

We drop the sum over the structure constants to avoid clutter. The denominator is symmetric in the interchange of $k^{b c}$ and $r^{c a}$ [42], so we can add the two terms, giving $k_{\mu}^{b c}+r_{\mu}^{c a} \sim p_{\mu}^{a b}$, which vanishes when contracted with $T_{\mu \nu}\left(p^{a b}\right)$.

The third term $\sim\left(r^{c a}\right)^{2}$ is

$$
\sim \sum_{k} \frac{1}{\left(\left(k^{b c}\right)^{2}\right)^{2}} k_{\mu}^{b c} T_{\mu \nu}\left(p^{a b}\right)
$$

When $\mu=i$ is spatial the integral is odd in $k_{i}$ and vanishes. The integral is nonzero when $\mu=0$, proportional to 
$\theta^{b}-\theta^{c}$. At zero momentum, though, $T_{00}=\left(\theta^{a}-\theta^{b}\right)^{2} \times$ $(1-1)=0$, while $T_{0 i} \sim\left(\theta^{a}-\theta^{b}\right) p_{i}$.

In the limit of zero momentum, $p_{0}=p_{i}=0$, the first term $\sim\left(p^{a b}\right)^{2}$ is

$$
\sim\left(\theta^{a}-\theta^{b}\right)^{2} \bigvee_{k} \frac{1}{\left(\left(k^{b c}\right)^{2}\right)^{2}\left(k^{c a}\right)^{2}} k_{\mu}^{b c} T_{\mu \nu}\left(p^{a b}\right) .
$$

The analysis is as for the third term. When $p_{i}=0$, the integral is nonzero only if $\mu=0$. For $\nu=0$, $T_{00}(0)=0$, while for $\nu=i, T_{0 i} \sim\left(\theta^{a}-\theta^{b}\right) p_{i}=0$ when $p_{i}=0$.

In conclusion, while there are extra contributions to the gluon self-energy when $\xi \neq 1$, the transverse condition on the gluon self-energy of Eq. (54) remains valid at zero momentum,

$$
\left(\theta^{a}-\theta^{b}\right) \Pi_{\mathrm{total} ; 0 \nu}^{a b, c d}\left(T\left(\theta^{a}-\theta^{b}\right), \mathbf{0}\right)=0 ; \quad a \neq b .
$$

This is valid for all $\theta_{a}$. When $\nu=0$, this shows that the Debye mass vanishes for off-diagonal elements, $a \neq b$, when $\theta^{a} \neq \theta^{b}$. This is a remarkable result. For spatial $\nu=i$, $\Pi_{\text {total } 0, i}^{a b, c d}\left(T\left(\theta^{a}-\theta^{b}\right), \mathbf{0}\right)$ vanishes, as $\Pi^{a b, c d}(0, \mathbf{0})_{0 i}$ does for when all $\theta^{a}=0$.

In the static limit, diagonal elements of the gluon self-energy satisfy a Ward identity like that for zero holonomy,

$$
p^{i} \Pi_{\text {total } ; i \nu}^{a a, b b}(0, \boldsymbol{p})=0 .
$$

(Remember there is no summation over $a$ or $b$.) Thus diagonal elements of $\Pi_{\text {total; } 00}^{a a, b b}(0,0)$ are nonzero, $\sim g^{2} T^{2}$, as the Debye mass for diagonal gluons.

A stronger constraint follows at arbitrary momentum. Since Eq. (63) is $\sim k_{\mu}^{b c} T_{\mu \nu}\left(p^{a b}\right)$, it vanishes if we contract with $p_{\nu}^{a b}$, and so

$$
p_{\mu}^{a b} p_{\nu}^{a b} \Pi_{\mathrm{total} ; \mu \nu}^{a b, c d}\left(p^{a b}\right)=0 .
$$

This is valid at arbitrary momentum and gauge fixing parameter, $\xi$ [8]. Notice that in order to obtain this relation, the contribution of the constraint to the gluon self-energy is essential, Eq. (51).

\section{F. Constraints with double traces}

In this section we show that the same results hold when the constraint is an arbitrary function of double traces,

$$
\mathcal{S}_{\text {cons }}=i \epsilon\left(B(\Theta)-\int \frac{d^{3} x}{V} \sum_{r=1}^{\infty} c_{r}\left|\operatorname{tr} \mathbf{L}^{r}(x)\right|^{2}\right),
$$

where $B(\Theta)$ is manifestly $Z(N)$ invariant. Consequently, if we choose one $\bar{A}$ to satisfy the constraint, there will be $N$ equivalent vacua which also satisfy the constraint. This does not preclude us from introducing such a constraint; we do so because in constructing effective theories, it is natural to use terms which are $Z(N)$ invariant.

Adding this to the action, instead of Eq. (23) the equation of motion is

$$
\left(\frac{-i \epsilon^{\mathrm{cl}}}{V}\right) \sum_{r=1}^{\infty} \sum_{b=1}^{N} i c_{r} r\left(\mathrm{e}^{i r\left(\theta_{a}-\theta_{b}\right)}-\mathrm{e}^{-i r\left(\theta_{a}-\theta_{b}\right)}\right)=\frac{8 \pi T^{3}}{3} \sum_{b=1}^{N} B_{3}\left(\frac{\theta_{a}-\theta_{b}}{2 \pi}\right) .
$$

In addition to Eq. (43), we also need

$$
\operatorname{tr}\left(\mathbf{L}_{2}^{r}(x)\right)_{\text {off }}^{\dagger}=+\frac{g^{2}}{2 i} \sum_{a \neq b=1}^{N} r\left(\mathrm{e}^{-i r \theta_{a}}-\mathrm{e}^{-i r \theta_{b}}\right) T \sum_{n=-\infty}^{+\infty} \frac{1}{p_{0}^{a b}}\left(\mathcal{A}_{0}^{b a}\left(x, p_{0}\right) \mathcal{A}_{0}^{a b}\left(x,-p_{0}\right)\right),
$$

thus at quadratic order the contribution of off-diagonal elements to the Lagrangian is

$$
\begin{aligned}
& -\left(\frac{-i \epsilon^{\mathrm{cl}}}{V}\right) \frac{g^{2}}{2 i} \sum_{r=1}^{\infty} c_{r} r \sum_{a \neq b=1, c=1}^{N}\left(\mathrm{e}^{i r\left(\theta_{a}-\theta_{c}\right)}-\mathrm{e}^{-i r\left(\theta_{a}-\theta_{c}\right)}-\mathrm{e}^{i r\left(\theta_{b}-\theta_{c}\right)}+\mathrm{e}^{-i r\left(\theta_{c}-\theta_{c}\right)}\right) \\
& \quad \times T \sum_{n=-\infty}^{+\infty} \frac{1}{p_{0}^{a b}}\left(\mathcal{A}_{0}^{b a}\left(x, p_{0}\right) \mathcal{A}_{0}^{a b}\left(x,-p_{0}\right)\right) .
\end{aligned}
$$

By using the equation of motion in Eq. (72), though, this reduces identically to the result of Eq. (48). Thus all of the results obtained previously by constraining terms linear in Polykov loops go through unchanged. This includes the identity of the free energy to $\sim g^{2}$ and the transversity of the gluon self-energy.
As seen previously for a constraint involves linear powers of the Polyakov loop, which was independent of the $\mathcal{J}_{r}$, for constraints with double traces, the gluon self-energy is independent of the specific coefficients that enter, the $c_{r}$.

Polyakov loops from constraints (or sources) also contribute to correlation functions of $\mathcal{A}_{0}$ to higher order. 
For example, cubic terms will involve two and three factors of $1 / p_{0}^{a b}$; assuming that the later cancel, as for the quadratic terms, the same reduction by the equations of motion appears plausible. It is natural to suppose that these will cancel other terms which arise from purely perturbative computations to $\sim g^{3}$, etc., but we have not explicitly verified this.

We also suggest that similar properties hold for arbitrary functions of Polyakov loops, but the above suffices for our purposes herein. Indeed, the generality of these results hints that a more general property of path ordered loops is at work, which is at present obscure to us.

\section{FREE ENERGY TO $\sim g^{3}$}

In describing the transition to a confined phase, with $\mathcal{A}_{0} \sim \Theta T / g$, when $\left|\theta_{a}-\theta_{b}\right| \sim 1$ for all $a$ and $b$, we can term the $\theta_{a}$ as "hard." Naively, the background field acts as an infrared cutoff for the off-diagonal modes. Of course this still leaves infrared divergences from the diagonal modes. (For quantities like the surface tension, some off-diagonal $\theta_{a b}$ vanish per se and contribute infrared divergences $[18,19]$.

In perturbation theory, it is well known that the static modes are infrared divergent, and contribute to the free energy at $\sim g^{3}$. We next consider how a "soft" background field, where $\left|\theta_{a}-\theta_{b}\right| \sim g$, contributes to the free energy. This describes how a holonomous plasma first emerges from the strict perturbative limit.

If the total self-energy at $\sim g^{2}$ is $\Pi_{\text {total } ; \mu \nu}^{a b, c d}\left(p^{a b}\right)$, then by resumming the ring diagrams, they contribute to the free energy

$$
\begin{aligned}
\mathcal{F}_{3}= & -\sum_{a, b=1}^{N} \mathcal{Y}_{p} \operatorname{tr} \log \left(\left(p^{a b}\right)^{2} \delta^{\mu \nu}\right. \\
& \left.+\left(\xi^{-1}-1\right) p_{\mu}^{a b} p_{\nu}^{a b}-\Pi_{\text {total } ; \mu \nu}^{a b, b a}\left(p^{a b}\right)\right),
\end{aligned}
$$

with the notation of Eq. (57). To obtain $\mathcal{F}_{3}$ we need to drop two contributions: that when $\Pi_{\text {total } ; \mu \nu}^{a b, c}\left(p^{a b}\right)=0$, as that is already included in the free energy to $\sim 1$, and that to linear order in $\Pi_{\text {total } ; \mu \nu}^{a b, c}\left(p^{a b}\right)$, as that is part of the free energy to $\sim g^{2}$. The derivative of $\mathcal{F}_{3}$ with respect to $\xi$ is proportional to

$$
\sim \xi^{-1} p_{\mu}^{a b} p_{\nu}^{a b} \Pi_{\mathrm{total} ; \mu \nu}^{a b, b a}\left(p^{a b}\right)
$$

and vanishes by Eq. (70).

In the perturbative vacuum, the computation of $\mathcal{F}_{3}$ is familiar [2]. The most infrared divergent term is from the static mode, with $p_{0}=0$. In this limit, the only component of $\Pi_{\mathrm{total} ; \mu \nu}^{a b, c d}\left(p^{a b}\right)$ which is nonzero is

$$
\Pi_{00}\left(p_{0}=0, \boldsymbol{p} \rightarrow 0\right)=m_{\text {Debye }}^{2}=\frac{g^{2} N}{3} T^{2},
$$

where $m_{\text {Debye }}^{2}$ is the Debye mass squared. Integrating over $\boldsymbol{p}$ with $\xi=1$,

$\mathcal{F}_{3}=-\sum_{a, b=1}^{N} \mathcal{Y}_{p} \operatorname{tr} \log \left(\boldsymbol{p}^{2}+m_{\text {Debye }}^{2}\right) \sim T m_{\text {Debye }}^{3} \sim g^{3} T^{4}$.

At weak but nonzero holonomy it is less straightforward computing $\mathcal{F}_{3}$. In perturbation theory we distinguish between diagonal gluons, with $a=b$, and off-diagonal gluons, with $a \neq b$. This separation is gauge dependent, but as for the free energy to $\sim 1$ and $\sim g^{2}$, we explicitly demonstrate the gauge invariance of the free energy to $\sim g^{3}$. Computation to higher order, $\sim g^{4}$, is deferred to future analysis [23].

\section{A. Off-diagonal gluons}

The computation of the self-energy to one loop order is given in Ref. [24]. Here we give an alternate derivation, using results from the hard thermal loop (HTL) limit. Typically, the HTL is computed after analytically continuing the Euclidean energy $p_{0} \rightarrow-i \omega$; it is valid for soft momenta, taking both $\omega$ and $|\boldsymbol{p}|$ soft, $\sim g T$.

In the Euclidean theory, for the colored momenta $p_{0}^{a b}=$ $p_{0}+T\left(\theta_{a}-\theta_{b}\right)$ to be soft requires that $p_{0}=0$ and that all $\theta_{a} \sim g$. In the HTL limit, the gluon self-energy is [26]

$$
\begin{aligned}
\Pi_{\text {pert } \mu \mu \nu}^{a b, c d}\left(p^{a b}\right) \approx & -\mathcal{K}_{\text {pert }}^{a b, c d}(\Theta) \delta \Gamma^{\mu \nu}\left(p^{a b}\right) \\
& -\left(m_{\text {pert }}^{2}\right)^{a b, c d}(\Theta) \delta \Pi^{\mu \nu}\left(p^{a b}\right) .
\end{aligned}
$$

This result is independent both of the gauge fixing parameter, and of the particular gauge chosen. The only requirement is that the external momenta are all soft.

The first term involves the function $\mathcal{K}$, which depends only upon the $\theta_{a}$ 's:

$$
\begin{aligned}
\mathcal{K}_{\text {pert }}^{a b, c d}(\Theta)= & \frac{4 \pi i}{3} g^{2} T^{3}\left(\delta ^ { a d } \delta ^ { b c } \sum _ { e = 1 } ^ { N } \left(B_{3}\left(\frac{\theta_{a}-\theta_{e}}{2 \pi}\right)\right.\right. \\
& \left.\left.+B_{3}\left(\frac{\theta_{e}-\theta_{b}}{2 \pi}\right)\right)-2 \delta^{a b} \delta^{c d} B_{3}\left(\frac{\theta_{a}-\theta_{c}}{2 \pi}\right)\right)
\end{aligned}
$$

in Ref. [26], $\mathcal{A}_{0}(T \theta)=2 B_{3}(\theta /(2 \pi)$ ) was used. (This corrects Eq. (158) of Ref. [26], where the coefficient on the right-hand side should be $2 \pi i g^{2} T^{3} / 3$ instead of $2 g^{2} T^{3}$.) The soft momenta enter through the function

$$
\delta \Gamma^{\mu \nu}\left(p^{a b}\right)=-\frac{1}{i p_{0}^{a b}} \delta \Pi^{\mu \nu}\left(p^{a b}\right)-u^{\mu} u^{\nu} \frac{1}{i p_{0}^{a b}} .
$$

$\delta \Pi^{\mu \nu}(p)$ is the standard function which appears in hard thermal loops, 


$$
\delta \Pi^{\mu \nu}(p)=-u^{\mu} u^{\nu}-i p_{0} \int \frac{d \Omega}{4 \pi} \frac{\hat{K}^{\mu} \hat{K}^{\nu}}{p \cdot \hat{K}} .
$$

The integral is over all directions of the unit spatial vector $\hat{k}$; $\hat{K}=(-i, \hat{k})$ is a null vector, $\hat{K}^{2}=0$. This function remains valid if $p_{0} \rightarrow p_{0}^{a b}=T\left(\theta_{a}-\theta_{b}\right)$.

The Debye mass squared for $\theta_{a} \neq 0$ also enters,

$$
\begin{aligned}
& \left(m_{\mathrm{pert}}^{2}\right)^{a b, c d}(\Theta) \\
& =g^{2} T^{2}\left(\delta^{a d} \delta^{b c} \sum_{e=1}^{N}\left(B_{2}\left(\frac{\theta_{a}-\theta_{e}}{2 \pi}\right)+B_{2}\left(\frac{\theta_{e}-\theta_{b}}{2 \pi}\right)\right)\right. \\
& \left.\quad-2 \delta^{a b} \delta^{c d} B_{2}\left(\frac{\theta_{a}-\theta_{c}}{2 \pi}\right)\right)
\end{aligned}
$$

in [26], $\mathcal{A}(T \theta)=6 B_{2}(\theta /(2 \pi))$ was used.

After analytic continuation, the above expressions apply for soft $\omega$ and $p$, and arbitrary $\theta_{a} \sim 1$. To compute $\mathcal{F}_{3}$, we need the limit in which $p_{0}=0$ and all $\theta_{a} \sim g$. In this limit, we can approximate $B_{2}(0)=1 / 6$, and $B_{3}(x) \approx x / 2$. In $\delta \Gamma^{\mu \nu}$, the term $2 \pi i B_{3}\left(\left(\theta_{a}-\theta_{b}\right) /(2 \pi)\right) / i p_{0}^{a b} \sim 1 / 2$ at small $\theta_{a}$. Doing so, we find that all terms $\sim \delta \Pi^{\mu \nu}\left(p^{a b}\right)$ cancel identically. This only leaves the term $\sim-u^{\mu} u^{\nu} /\left(i p_{0}^{a b}\right)$ on the right-hand side of Eq. (81). However, this enters proportional to $B_{3}\left(\left(\theta_{a}-\theta_{e}\right) /(2 \pi)\right)+B_{3}\left(\left(\theta_{e}-\theta_{b}\right) /(2 \pi)\right)$. By the previous analysis in Eq. (51), this also cancels against the contribution of the constraint term, $\Pi_{\text {cons; } \mu \nu}$.

This implies that for small $\Theta$, all contributions to the selfenergy for off-diagonal gluons vanish for $p_{0}=0$ and $\Theta \sim g$. This cancellation only occurs for small $\Theta$, and does not hold when $\Theta \sim 1$.

This does not imply that there are long ranged fields. In the presence of the background $\bar{A}$ field, at leading order the inverse propagator for the transverse gluons is

$$
\Delta^{-1}=\left(p_{0}+T\left(\theta_{a}-\theta_{b}\right)\right)^{2}+\boldsymbol{p}^{2} .
$$

Thus even for static modes with $p_{0}=0$, a nonzero holonomy, $\theta_{a} \neq 0$, acts like a mass term.

The analysis implies that static electric fields are not screened for small $\Theta$. This can also be seen from the transversity of the total gluon self-energy, $\Pi_{\text {total } ; \mu \nu}^{a b, c d}\left(p^{a b}\right)$ in Eq. (54). In the static limit, as $\boldsymbol{p} \rightarrow 0$ this reduces to

$$
\left(\theta_{a}-\theta_{b}\right) \Pi_{\text {total; } 00}^{a b, c d}\left(2 \pi T\left(\theta_{a}-\theta_{b}\right), 0\right)=0 .
$$

Consequently, when $\theta_{a}-\theta_{b} \neq 0$, the self-energy vanishes, $\Pi_{\mathrm{total} ; 00}^{a b, c d}\left(T\left(\theta_{a}-\theta_{b}\right), 0\right)=0$. Clearly, for this to hold, it is essential that the gluon self-energy is transverse.

This is very different from when $\theta_{a}=0$; then going to the static limit does not constrain $\Pi_{00} \sim m_{\text {Debye }}^{2}$. From
Eq. (77), $m_{\text {Debye }}^{2} \neq 0$, so static electric fields are screened when $\theta_{a}=0$.

This behavior can be derived directly without explicit evaluation of the one loop diagrams. We use the expressions for the hard thermal loops in a holonomous plasma [26]. This only applies for soft momenta, so both the spatial momentum $p$ and the $\theta_{a}$ are soft, $\sim g$. Consider the diagram with two three gluon vertices. After summing over the loop momentum $k_{0}$, from Eqs. (115) and (116) of Ref. [26] the contribution to $\Pi^{i j}$ is proportional to

$$
\mathcal{J}^{i j}\left(p, T \theta_{1}, T \theta_{2}\right) \sim \int_{0}^{\infty} d^{3} k \frac{k^{i} k^{j}}{E_{k} E_{p-k}} \int \frac{d \Omega}{4 \pi}\left(\mathcal{I}_{2}+\mathcal{I}_{3}\right) .
$$

We assume that the loop momentum $k$ is hard, so in each three gluon vertex we can take $\sim k^{i}$, dropping terms $\sim p^{i}$. Similarly, we approximate $E_{p-k} \sim k$. Then the momentum dependence arises entirely from the statistical distribution functions and from the energy denominators. This is given by

$$
\begin{aligned}
& \mathcal{I}_{2}=\frac{n\left(E_{k}-i T \theta_{1}\right)-n\left(E_{p-k}+i T \theta_{2}\right)}{i p_{0}^{12}-E_{k}+E_{p-k}} ; \\
& \mathcal{I}_{3}=\frac{n\left(E_{p-k}-i T \theta_{2}\right)-n\left(E_{k}+i T \theta_{1}\right)}{i p_{0}^{12}+E_{k}-E_{p-k}},
\end{aligned}
$$

where $p_{0}^{12}=p_{0}+T\left(\theta_{1}+\theta_{2}\right)$.

These factors arise from Landau damping, and involve a difference of hard energies. The difference is a soft energy, so we need to expand $E_{p-k} \approx k-\hat{k} \cdot \boldsymbol{p}+\ldots$ Since we are computing the self-energy for Euclidean momentum, we work in the static limit, $p_{0}=0$. For simplicity we assume $\theta_{1}=0$ and $\theta_{2}=\theta$. Under these approximations,

$\mathcal{I}_{2} \approx \frac{1}{-\hat{k} \cdot \boldsymbol{p}+i T \theta}(n(k)-n(k-\hat{k} \cdot \boldsymbol{p}+i T \theta)) \approx-\frac{d}{d k} n(k)$.

and

$\mathcal{I}_{3} \approx \frac{1}{\hat{k} \cdot \boldsymbol{p}+i T \theta}(n(k-\hat{k} \cdot \boldsymbol{p}-i T \theta)-n(k)) \approx-\frac{d}{d k} n(k)$.

Each term is nonzero, but the point is that it is independent of both the external spatial momentum, $\boldsymbol{p}$, and the holonomy, $T \theta$. This is most unexpected, as it is certainly possible for the result to depend upon the dimensionless ratio $|\boldsymbol{p}| /(\theta T)$.

It is also useful to consider the behavior of the free energy. At one loop order any mode with nonzero energy, 
$p_{0} \neq 0$, clearly contributions to the determinant, $\operatorname{tr} \log \Delta^{-1}$, are regular about $\theta_{a}=0$. Thus modes with nonzero energy contribute only to the terms quadratic and quartic in the $\theta_{a}$ 's:, $\sim\left(\theta_{a}-\theta_{b}\right)^{2}$ and $\sim\left(\left(\theta_{a}-\theta_{b}\right)^{2}\right)^{2}$ in $B_{4}\left(\left(\theta_{a}-\theta_{b}\right) /(2 \pi)\right)$, Eqs. (10) and (11). There is also a cubic term in $B_{4}\left(\left(\theta_{a}-\theta_{b}\right) /(2 \pi)\right), \sim\left(\left(\theta_{a}-\theta_{b}\right)^{2}\right)^{3 / 2}$; it is easy to show that this arises uniquely from the mode with static energy, $p_{0}=0$. Thus the origin of the cubic term in the one loop potential is similar as that of $\mathcal{F}_{3}$ when $\theta_{a}=0$, Eq. (78). When the $\theta_{a}$ are soft, this cubic term at one loop order is $\sim g^{3}$, like that in perturbation theory. Similarly, those at two loop order are $\sim g^{5}$. What is unexpected is that the free energy does not appear to be continuous as $\Theta \rightarrow 0$ : there are cubic terms $\sim\left|\theta_{a}\right|^{3}$ when $\theta_{a} \neq 0$, but these vanish as $\theta_{a} \rightarrow 0$. In contrast, at zero holonomy there is a cubic term $\sim g^{3}$.

\section{B. Diagonal elements to $\sim g^{3}$}

In principle, the computation of the contribution of color diagonal gluons to the free energy at weak holonomy is straightforward. As argued previously, gauge invariant sources must be used, minimized with respect to the background field, and the Debye masses in the presence of the background field computed.

We show that when the explicit potentials are computed, that a surprise arises. Because the potential at one loop order involves a sum over an infinite number of loops, any source must also involve an infinite sum, of a specific form.

Our arguments can be made precise for two and an infinite number of colors. After treating these two examples in detail, we discuss arbitrary $N$.

\section{Two colors}

For two colors, define $\theta_{1}=-\theta_{2}=\pi q$. To one loop order the perturbative potential is

$$
\mathcal{V}_{1}(q)=\pi^{2} T^{4}\left(-\frac{1}{15}+\frac{4}{3} q^{2}(1-q)^{2}\right) .
$$

We set $T=1$ for convenience. Normalized to unity, the Polyakov loop $\ell=\cos (\pi q)$, with $q=0$ the perturbative vacuum, and $q=1 / 2$ the confined. We add two sources,

$$
\mathcal{V}_{j}(q)=4 j_{1}\left(\ell^{2}-1\right)+16 j_{2}\left(\ell^{4}-1\right)
$$

The potential with just $j_{1}$ was considered in Ref. [27]; that with $j_{1}$ and $j_{2}$ was discussed in Ref. [28]. The total potential is then

$$
\mathcal{V}_{\text {tot }}(q)=\mathcal{V}_{1}(q)+\mathcal{V}_{j}(q)
$$

For large values of $j_{1}$ and $j_{2}$ the potential minimizes the loop, and drives the theory to the confined vacuum, $q=1 / 2$. Our interest is how this occurs.
Begin with $j_{2}=0$. As $j_{1}$ increases, there is a transition from $q=0$ to $q \neq 0$ at

$$
j_{1}^{0}=\frac{\pi^{2}}{48}, \quad j_{2}=0 .
$$

This transition is of first order, directly to the confining vacuum with $q=1 / 2[27,28]$.

This is not what we require, however, but rather a transition to a nonzero but arbitrarily small value of $q \neq 0$. Consider expanding about the confined phase, with $q=1 / 2$ :

$$
\begin{aligned}
\mathcal{V}_{\text {tot }}\left(\frac{1}{2}-\delta q\right) \approx & \frac{\pi^{2}}{12}-4 j_{1}-16 j_{2}+4 \pi^{2}\left(j_{1}-\frac{1}{6}\right) \delta \theta^{2} \\
& +\frac{4}{3} \pi^{4}\left(-\frac{1}{\pi^{2}}+j_{1}-12 j_{2}\right) \delta \theta^{4}+\cdots
\end{aligned}
$$

Thus there is a line of second order transitions from the deconfined to the confined phase when $j_{1}=1 / 6$.

For the quartic coupling to be positive [28],

$$
j_{2} \geq \frac{1}{12}\left(\frac{1}{6}-\frac{1}{\pi^{2}}\right)
$$

This is not sufficient: at $j_{1}=1 / 6$ and this value of $j_{2}$, the value of the potential at $q=1 / 2$ is higher than at $q=0$, not lower.

Fix $j_{1}=1 / 6$, and move up in $j_{2}$ to

$$
j_{1}^{\text {crit }}=\frac{1}{6}, \quad j_{2}^{\text {crit }}=\frac{1}{16}\left(\frac{\pi^{2}}{12}-\frac{2}{3}\right) .
$$

At this point, the potential has an uncommon form, illustrated in Fig. 1. The value of the potential is equal

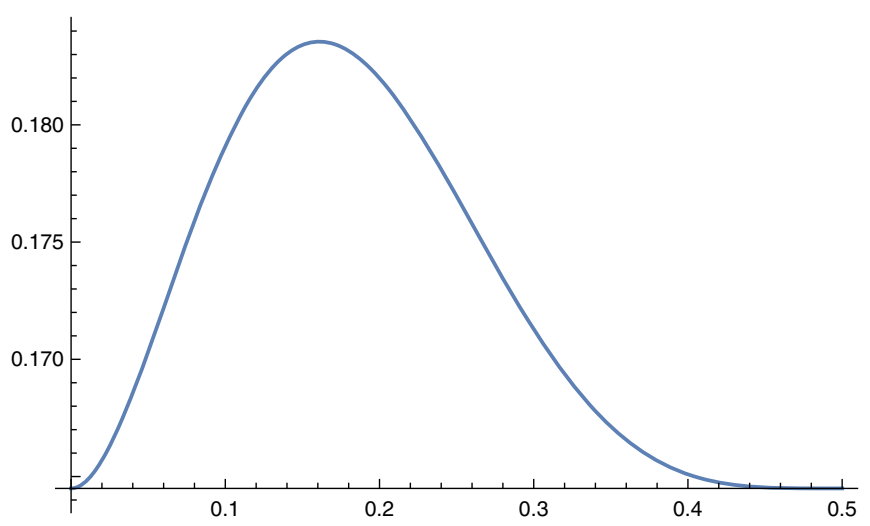

FIG. 1. A plot of the potential for the critical first order point, Eq. (96). The masses squared is nonzero about the perturbative vacuum, $q=0$, but vanishes about the confining vacuum, $q=1 / 2$. 
at $q=0$ and $q=1 / 2$, with a barrier between them, and so the transition is of first order. Nevertheless, the mass in the confining vacuum, at $q=1 / 2$, vanishes. We call this a critical first order transition; they also occur at infinite $N$ in some matrix models [29,30]. It occurs for two colors because the potential is not just a simple polynomial in $q$.

Moving up in $j_{2}$ for a constant value of $j_{1}=1 / 6$, there is a standard second order transition. Now consider first increasing $j_{1}$ from $j_{1}^{\text {crit }}$. From Eq. (94), the potential at $q=1 / 2$ vanishes along the straight line

$$
j_{2}=\frac{1}{4}\left(j_{1}^{0}-j_{1}\right)
$$

Along this line, there is a first order transition from $q=0$ directly to the confining vacuum, $q=1 / 2$.

The behavior for $j_{1}<j_{1}^{\text {crit }}$ is more involved. In this case we take

$$
j_{1}=j_{1}^{\text {crit }}-\delta j_{1}, \quad j_{2}=\frac{1}{4} \delta j_{1}+\delta j_{2}
$$

Expanding about this point, we find a first order transition from $q=0$ to $q=1 / 2-\delta q$,

$$
(\delta q)^{2}=8 a \delta j_{1}, \quad \delta j_{2}=-a \delta j_{1}^{2}, \quad a=\frac{9 \pi^{2}}{3 \pi^{4}+16} .
$$

That $\delta q \sim \sqrt{\delta j_{1}}$, instead of $\delta q \sim \delta j_{1}$, follows because the mass vanishes about the confined phase at $j_{1}^{\text {crit }}$.

Thus there is a line of first order transitions as $j_{1}$ decreases. Along this line, there is a first order transition from $q=0$ to a value of $q_{0}<1 / 2$. This line goes down to $j_{1}=0$, where there is a first order transition at $j_{1}=0$ and $j_{2}^{0} \approx 0.03615 \ldots ;$ at this point, the minimum of the potential jumps from $q=0$ to $q_{0} \approx 0.145 \ldots$

This gives rise to the phase diagram of Fig. 2. There is an unbroken line of first order transitions, with no smooth transition from $\langle q\rangle=0$ to a nonzero value. This phase diagram is qualitatively different form Fig. 1 in Ref. [28], where the line of first order transitions terminates at $j_{1}^{\text {crit }}$.

Thus the two sources used in Eq. (91) are not adequate to generate a small value of $\Theta$ for arbitrarily small sources. Consider the expansion for small $q$. Then the sources, as functions of $\cos (\pi q)$, begin at quadratic order. The same is true for the potential at one loop order, but in addition, there is a term of cubic order, with a negative sign. The terms from the sources can be tuned so that the coefficient of the quadratic term vanishes, but that still leaves negative cubic term, which drives a first order transition.

This argument is unavoidable for two colors, and can be immediately generalized to three colors. We comment that the appearance of a cubic term, which implies nonanalyticity in $q$, is because the potential involves a sum over an infinite number of loops.

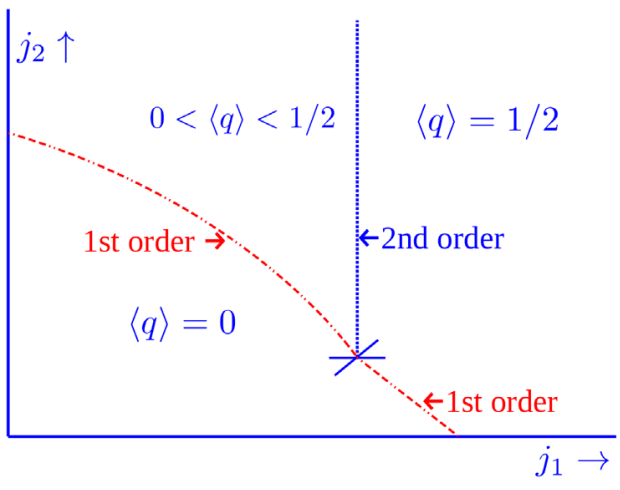

FIG. 2. The phase diagram for the effective model for two colors, with the potential of Eq. (94). There are three regions: strict perturbative, with $\left\langle q_{a}\right\rangle=0$; holonomous plasma, with $0<\left\langle q_{a}\right\rangle<1 / 2$, and confined, with $\left\langle q_{a}\right\rangle=1 / 2$. The cross denotes $\left(j_{1}^{\text {crit }}, j_{2}^{\text {crit }}\right)$, Eq. (96). Note that there is an unbroken line of first order transitions between the confined and perturbative phases.

\section{Infinite colors}

For four or more colors, there is more than one independent $\theta_{a}$. While the presence of a cubic term in the perturbative potential, $B_{4}\left(\theta_{a}-\theta_{b}\right)$, suggests that one cannot smoothly move from $\theta_{a}=0$ to nonzero $\theta_{a}$, it is not evident that it might not happen for one special direction of the $\theta_{a}$.

In this subsection we compute for an infinite number of colors, using standard techniques for matrix models at large $N[29,32,33,43]$, and in particular using the known solution for this particular model [30,31]. We revert to using the $\theta_{a}$, as in Refs. [30,31] At large $N$ we replace the discrete label $a$ by a continuous index $x$, where $x=a / N-1 / 2$, and introduce the eigenvalue density,

$$
\rho(\theta)=\frac{d x}{d \theta}
$$

At large $N$ the one loop potential is $N^{2}$ times

$$
\begin{aligned}
\mathcal{V}_{1}(\theta) \sim & \int_{-\pi}^{\pi} d \theta_{1} \int_{-\pi}^{\pi} d \theta_{2} \rho\left(\theta_{1}\right) \rho\left(\theta_{2}\right) \\
& \times\left|\theta_{1}-\theta_{2}\right|^{2}\left(1-\frac{\left|\theta_{1}-\theta_{2}\right|}{2 \pi}\right)^{2},
\end{aligned}
$$

up to an overall constant, Eq. (10). For small $\theta$ we certainly expect a cubic term of negative sign, but to establish this definitively requires the explicit solution for the eigenvalue density [31]. Previous study concentrated on the transition from the confined to the deconfined phase, but the analysis can be adapted to how the theory leaves the perturbative limit.

In terms of the eigenvalue density the $n$th Polyakov loop equals 


$$
\ell_{n}=\frac{1}{N} \operatorname{tr} \mathbf{L}^{n}=\int_{-\pi}^{\pi} d \theta \rho(\theta) \mathrm{e}^{i n \theta}
$$

We assume that by an overall $Z(N)$ rotation the expectation value of all loops is real, so the eigenvalue density $\rho(\theta)$ is an even function in $\theta$.

The perturbative potential can be rewritten in a power series in Polyakov loops, Eq. (10). Notice that the overall sign is negative, so the potential is minimized when all loops are maximal: all $\ell_{n}=1$, so all $\theta_{a}(x)=0$.

In studying the transition from the confined to the deconfined phase, Ref. [31] assumed that the coefficient of Eq. (10) is positive. The potential is then minimized when all loops vanish, which is the confined phase.

The eigenvalue density for large $N$ is soluble regardless of the overall sign of Eq. (10). In the notation of Ref. [31], the solution is the case $s=4$. While the effective potential is a function of all $\ell_{n}$, we can integrate out all loops except for the first, $\ell_{1}$. It is necessary to introduce an external field for $\ell_{1}, \omega$. The potential for $\omega$ is

$$
\mathcal{F}_{1}(\omega)=+2 \int_{0}^{\omega} d \omega^{\prime} \ell_{1}\left(\omega^{\prime}\right) .
$$

The sign on the right-hand side is positive, opposite to the negative sign in Eq. (31) of Ref. [31]. This is due to overall change in sign of the potential in Eq. (10).
The solution for the eigenvalue density is [31]

$$
\begin{aligned}
\rho(\theta)= & \frac{1}{2 \pi}\left(\left(\pi-\theta_{0}-2 \omega \sin \theta_{0}\right)\left(\delta\left(\theta-\theta_{0}\right)+\delta\left(\theta+\theta_{0}\right)\right)\right. \\
& +1+2 \omega \cos \theta) .
\end{aligned}
$$

The is defined for $|\theta| \leq \theta_{0}$, with two $\delta$-function singularities at each end, for $\theta= \pm \theta_{0}$. The solution vanishes when $|\theta|>\theta_{0}$, which we call a single gap (as $\rho(\theta)$ is even in $\left.\theta\right)$. The eigenvalue density can be computed for $s=1,2,3$, and 4 , but the singularities at $\theta= \pm \theta_{0}$ are special to $s=4[31]$.

The endpoint of the gap, $\theta_{0}$, is related to the background field, $\omega$, through the relation

$$
\omega=\frac{1}{6}\left(\frac{\left(\pi-\theta_{0}\right)^{3}}{\sin \theta_{0}+\left(\pi-\theta_{0}\right) \cos \theta_{0}}\right) .
$$

The strict perturbative limit is when $\theta_{0}=0$, as

$$
\rho_{\text {pert }}(\theta)=\delta(\theta) ; \quad \omega=\omega_{c}=\frac{\pi^{2}}{6} .
$$

Expanding in $\omega$,

$$
\omega=\frac{\pi^{2}}{6}-\delta \omega,
$$

the solution of Eq. (105) is

$$
\theta_{0}(\omega)=\frac{2}{\pi}\left(\delta \omega+\frac{1}{3}\left(1+\frac{6}{\pi^{2}}\right) \delta \omega^{2}+\frac{2}{9}\left(1+\frac{1}{\pi^{2}}+\frac{30}{\pi^{4}}\right) \delta \omega^{3}+\frac{5}{27}\left(1-\frac{1}{\pi^{2}}+\frac{144}{\pi^{6}}\right) \delta \omega^{4}+\cdots\right)
$$

With the eigenvalue density of Eq. (104), the first Polyakov loop equals

$$
\ell_{1}(\omega)=\frac{1}{\pi}\left(\omega \theta_{0}+\sin \theta_{0}+\cos \theta_{0}\left(\pi-\theta_{0}-\omega \sin \theta_{0}\right)\right) .
$$

Loops with $n \geq 2$ are given by

$$
\ell_{n \geq 2}=\frac{1}{\pi}\left(\left(\pi-\theta_{0}\right) \cos \left(n \theta_{0}\right)+\frac{\sin \left(n \theta_{0}\right)}{n}+\frac{2 \omega n}{n^{2}-1}\left(-n \sin \theta_{0} \cos \left(n \theta_{0}\right)+\cos \theta_{0} \sin \left(n \theta_{0}\right)\right) .\right.
$$

We need to compute these loops not as functions of $\theta_{0}$, but of the external field, $\omega$. For $\ell_{1}$,

$$
\ell_{1}(\delta \omega) \approx 1-b_{2} \delta \omega^{2}-b_{3} \delta \omega^{3}-b_{4} \delta \omega^{4}+\cdots
$$

where

$$
b_{2}=\frac{2}{\pi^{2}}, \quad b_{3}=\frac{4}{9 \pi^{2}}\left(1+\frac{12}{\pi^{2}}\right), \quad b_{4}=\frac{2}{9 \pi^{2}}\left(1+\frac{1}{\pi^{2}}+\frac{84}{\pi^{2}}\right)
$$

Solving for $\mathcal{F}_{1}$ from Eqs. (103),

$$
\mathcal{F}_{1}(\omega)-\mathcal{F}_{1}\left(\omega_{c}\right)=2\left(\delta \omega-\frac{b_{2}}{3} \delta \omega^{3}-\frac{b_{3}}{4} \delta \omega^{4}+\cdots\right)
$$


The potential, as a function of $\ell_{1}$, is given by

$$
\mathcal{V}_{1}\left(\ell_{1}\right)-\mathcal{V}_{1}(1)=\mathcal{F}_{1}(\omega)+\left.2 \omega \ell_{1}(\omega)\right|_{\omega=\omega\left(\ell_{1}\right)}-\left(\mathcal{F}_{1}\left(\omega_{c}\right)+2 \omega_{c}\right)
$$

As a function of $\omega$,

$$
\mathcal{V}_{1}\left(\ell_{1}\right)-\mathcal{V}_{1}(1) \approx \frac{2}{3} \delta \omega^{2}+\frac{4}{27}\left(1-\frac{6}{\pi^{2}}\right) \delta \omega^{3}+\frac{2}{27}\left(-1+\frac{8}{\pi^{2}}+\frac{24}{\pi^{4}}\right) \delta \omega^{4}+\cdots
$$

The right-hand side is a function of $\delta \omega$, but it is necessary to invert Eq. (111) and write it as a function of $\ell_{1}$. We introduce

$$
(\delta \rho)^{2}=\frac{\pi^{2}}{2}\left(1-\ell_{1}\right)
$$

this $\delta \rho$ is, by definition, a measure of the deviation from zero for all eigenvalues. Equation (111) gives

$$
\delta \omega=\delta \rho-\frac{1}{9}\left(1+\frac{12}{\pi^{2}}\right) \delta \rho^{2}+\frac{2}{81}\left(-1+\frac{111}{4 \pi^{2}}-\frac{9}{\pi^{4}}\right) \delta \rho^{3}+\cdots .
$$

Substituting this into Eq. (115), we obtain

$$
\mathcal{V}_{1}\left(\ell_{1}\right)-\mathcal{V}_{1}(1) \approx \frac{2}{3}(\delta \rho)^{2}-\frac{8}{3 \pi^{2}}(\delta \rho)^{3}+\frac{2}{9 \pi^{2}}\left(1+\frac{12}{\pi^{2}}\right)(\delta \rho)^{4}+\cdots
$$

As a function of $\delta \omega$, the $n$th Polyakov loop of Eq. (110) is

$$
\ell_{n}(\delta \omega)=1-\frac{2}{\pi^{2}} n^{2}(\delta \omega)^{2}-\frac{4\left(12+\pi^{2}\right)}{9 \pi^{4}} n^{2}(\delta \omega)^{3}-\frac{2}{9 \pi^{6}}\left(84 n^{2}+\left(4-3 n^{2}\right) n^{2} \pi^{2}+n^{2} \pi^{4}\right)(\delta \omega)^{4}+\cdots
$$

Using Eq. (117),

$$
\ell_{n}\left(\ell_{1}\right) \approx 1-\frac{2}{\pi^{2}} n^{2}(\delta \rho)^{2}+\frac{2}{3 \pi^{4}} n^{2}\left(n^{2}-1\right)(\delta \rho)^{4}+\cdots
$$

From Eq. (116), $\delta \rho$ is defined as the deviation of $\ell_{1}$ from unity, and so all terms of higher order in $\delta \rho$, vanish. This explains why the quartic term in $\ell_{1}$ vanishes. For any $n$, while there is a term cubic in $\delta \omega$ in Eq. (119), there is no term cubic in $\delta \rho$. This accords with the intuition that in expanding about the perturbative vacuum, that it is an expansion in even powers of the $\theta_{a}$, and hence of $\delta \rho$.

Consider adding a constraint (or source) to a general form for the effective potential of $\ell_{1}$,

$$
\mathcal{V}_{\mathrm{eff}}\left(\ell_{1}\right)=\mathcal{V}_{1}\left(\ell_{1}\right)+\sum_{n, m=1}^{\infty} c_{n}^{m} \ell_{n}^{2 m}
$$

The term quadratic in $\delta \rho$ vanishes when

$$
\sum_{n, m} c_{n}^{m} n^{2}=\frac{\pi^{2}}{6}
$$

At this point,

$$
\mathcal{V}_{\mathrm{eff}}\left(\ell_{1}\right)-\mathcal{V}_{\mathrm{eff}}(1) \approx-\frac{8}{3 \pi^{2}}(\delta \rho)^{3}+O\left(\delta \rho^{4}\right)
$$

Thus at the point where the term quadratic in $\delta \rho$ vanishes, there is a term cubic in $\delta \rho$, with negative sign. This implies that there is a transition of first order before this point is reached. The presence of a cubic term is nontrivial, as any single Polyakov loop does not have such as term, Eq. (120). It is the natural extension of the cubic term in the $\theta_{a}$ 's, expressed in terms of the correct eigenvalue density.

There is a caveat to the above. In adding terms proportional to the second or higher Polyakov loops, the eigenvalue densities sometimes develop solutions with two or more gaps [33]. We ignore this possibility, but it seems reasonable to suggest that even such multigap solutions will exhibit the first order transition above.

Consequently, as for the case with two colors, for any constraint with a finite number of Polyakov loops, there is a solid region of nonzero measure where the strict 
perturbative regime holds, with a first order transition to a holonomous plasma.

\section{Potentials}

In Sec. (IV B 1) we showed explicitly that a potential involving the two sources of Eq. (91) necessarily involves a first order transition. This is immediately generalized to any finite number of loops, due to the cubic term in the perturbative potential. We showed in the previous section that this remains valid for an infinite number of colors. It is natural to assume this is true for any $N$.

What is required is a source which is linear in $\Theta$ for small $\Theta$. Consider the Bernoulli polynomial,

$$
B_{2}(\Theta) \sim \sum_{n=1}^{\infty} \frac{1}{n^{2}}\left|\operatorname{tr} \mathbf{L}^{n}\right|^{2} .
$$

In this case, Eq. (122) naively diverges. The sum is given in Eq. (19), and has a term linear in the $\theta_{a}$ for small $\theta_{a}$.

This is true for any $B_{n}(x)$ when $n$ is odd. However, the odd $B_{n}(x)$ are also odd in $x$, and any term added to the action must be even in $x$. This suggests that $B_{2}(x)$ is a natural term to use either as a source, or as a nonperturbative potential in effective models [35-39]. Of course this does not imply that $B_{2}(x)$ must be used, only that any such potential must involve a sum over an infinite number of Polyakov loops, and have a term linear in $\theta_{a}$ about the origin.

\section{CONCLUSIONS}

In this paper we have considered the behavior of the free energy at nonzero holonomy in perturbation theory, and showed that they exhibit several unexpected features.

At one loop order, Sec. II, the free energy at nonzero holonomy is automatically gauge invariant. This is no longer true at two loop order, $\sim g^{2}$, Sec. III. The simplest way to ensure gauge invariance is by using sources which are themselves gauge invariant. As the thermal Wilson line is an $S U(N)$ matrix, it has $N-1$ eigenvalues which are gauge invariant. Thus one expects that it should suffice to use sources which are a sum over $N-1$ Polyakov loops.

To two loop order, the free energy is the same if computed by various other means, as long as it is expressed as a function of the eigenvalues of the thermal Wilson line. Since any Polyakov loop is a nonlinear function of the background field, these sources contribute not just to a term linear in quantum fluctuations, but to terms of quadratic and higher order. These quadratic terms are a novel contribution to the gluon self-energy, and are essential to ensuring that the gluon self-energy is transverse in the momentum, and so obeys the Ward identity of Eq. (70).

The new contributions to the gluon self-energy do not matter for the free energy to $\sim g^{2}$, but when the holonomy is weak, they do affect terms $\sim g^{3}$, Sec. IV. At this order, it is possible to separate between the contributions of offdiagonal and diagonal gluons.
The contribution of off-diagonal gluons is unusual. Typically, as a source is turned off, the contribution of the source to free energy vanishes smoothly. We show that this is not true for nonzero holonomy: while off-diagonal gluons contribute to $\sim g^{3}$ at zero holonomy, their contribution vanishes when the holonomy is small but nonzero, even infinitesimally. This was shown directly by computation in Ref. [24], and by using the methods of hard thermal loops in Sec. IV A. The latter has the advantage of being independent of the details of the computation, and so more general.

In future work the BRST identities are used to analyze the free energy to $\sim g^{4}$, and used to show that this contribution vanishes smoothly as the holonomy does [25]. This is reasonable, since for weak holonomy the $\sim g^{3}$ term from off-diagonal gluons is uniquely sensitive to the infrared physics, while terms $\sim g^{4}$ only probe strong holonomy, and are not sensitive to infrared divergences.

The contribution of diagonal gluons is also novel. Most sources are linear in the field, and so the change in the field is small when the source is. This is not true for nonzero holonomy, using sources which involve a finite number of Polyakov loops. By checking if an extremal point is in fact a minimum, we show that for two and an infinite number of colors, that sources which are a sum of any finite number of Polyakov loops do not suffice, and expect this remains true for any $N$. This analysis depends crucially upon the fact that in $3+1$ dimensions, at one loop order the free energy for nonzero holonomy has a cubic term with negative sign. Instead, to obtain a source which changes the field linearly for small values, it is necessary to sum over an infinite number of Polyakov loops. We present one form which suffices in Sec. IV B 3, which arises for a massless field in $1+1$ dimensions.

The discontinuity in the free energy to $\sim g^{3}$ is rather peculiar, and is not expected to arise if the holonomy is generated dynamically. Nonzero holonomy can be studied on a femto-torus [44], where the free energy is certainly continuous when the radius of the torus is small. Of course this does not exclude a discontinuity as the radius of the torus diverges, as then the semiclassical techniques which can be used for small radii do not apply.

In a future work the generation of holonomy by dynamical fields is studied in a simple toy model [45]. This is a theory of a massless, adjoint ghost field which lives on a $1+1$ dimensional manifold, which is embedded isotropically in $3+1$ dimensions. Using such a model, it is shown that the peculiarities found for static sources do not arise. If the holonomy is generated dynamically, then adding the contribution of off-diagonal ghosts to the free energy gives a result in which the free energy to $\sim g^{3}$ behaves smoothly as the holonomy vanishes. This accords with the prejudice that discontinuities should not arise in physical quantities.

This example also confirms the principal lesson of the present analysis. Which is that even apparently 
straightforward computations in perturbation theory, when done with care, can yield insight into such nonperturbative phenomenon as the generation of nonzero holonomy.

\section{ACKNOWLEDGMENTS}

R. D. P. is funded by the U.S. Department of Energy under Contract No. DE-SC0012704. H. N. was supported by the Special Postdoctoral Researchers program of RIKEN and the Japan Society for the Promotion of Science (JSPS) Grant-in-Aid for Scientific Research (KAKENHI) Grant No. 18H01217. V. S. is funded by the U.S. Department of Energy under Contract No. DESC0020081. C. P. K. A. thanks R. D. P. and BNL for their hospitality and support.
[1] E. V. Shuryak, Phys. Lett. B 78, 150 (1978); E. V. ShuryakYad. Fiz. 28, 796 (1978) [Sov. J. Nucl. Phys. 28, 408 (1978)].

[2] J. I. Kapusta, Nucl. Phys. B148, 461 (1979).

[3] T. Toimela, Phys. Lett. 124B, 407 (1983).

[4] P. B. Arnold and C.-X. Zhai, Phys. Rev. D 50, 7603 (1994); C.-x. Zhai and B. M. Kastening, Phys. Rev. D 52, 7232 (1995).

[5] K. Kajantie, M. Laine, K. Rummukainen, and Y. Schroder, Phys. Rev. D 67, 105008 (2003).

[6] N. Haque, M. G. Mustafa, and M. Strickland, Phys. Rev. D 87, 105007 (2013); N. Haque, J. O. Andersen, M. G. Mustafa, M. Strickland, and N. Su, Phys. Rev. D 89, 061701 (2014); J. O. Andersen, N. Haque, M. G. Mustafa, and M. Strickland, Phys. Rev. D 93, 054045 (2016).

[7] O. Philipsen, in 37th International Symposium on Lattice Field Theory (Lattice 2019) Wuhan, Hubei, China (2019), arXiv:1912.04827.

[8] D. J. Gross, R. D. Pisarski, and L. G. Yaffe, Rev. Mod. Phys. 53, 43 (1981).

[9] N. Weiss, Phys. Rev. D 24, 475 (1981).

[10] H. T. Elze, U. W. Heinz, K. Kajantie, and T. Toimela, Z. Phys. C 37, 305 (1988).

[11] K. Enqvist, K. Kajantie, L. Karkkainen, and K. Rummukainen, Phys. Lett. B 249, 107 (1990).

[12] V. M. Belyaev, Phys. Lett. B 254, 153 (1991).

[13] V. M. Belyaev, I. I. Kogan, G. W. Semenoff, and N. Weiss, Phys. Lett. B 277, 331 (1992); A. V. Smilga, Ann. Phys. (N.Y.) 234, 1 (1994); I. I. Kogan, Phys. Rev. D 49, 6799 (1994).

[14] T. Bhattacharya, A. Gocksch, C. Korthals Altes, and R. D. Pisarski, Phys. Rev. Lett. 66, 998 (1991); Nucl. Phys. B383, 497 (1992); H. Sawayanagi, Phys. Rev. D 45, 3823 (1992); Int. J. Mod. Phys. A 09, 4601 (1994).

[15] V. V. Skalozub, Phys. Rev. D 50, 1150 (1994).

[16] V. V. Skalozub, Mod. Phys. Lett. A 07, 2895 (1992); V. V. Skalozub and I. V. Chub, Yad. Fiz. 57, 344 (1994) [Phys. At. Nucl. 57, 324 (1994)]; V. V. Skalozub, Int. J. Mod. Phys. A 09, 4747 (1994); O. A. Borisenko, J. Bohacik, and V. V. Skalozub, Fortschr. Phys. 43, 301 (1995); V. V. Skalozub and A. V. Strelchenko, Eur. Phys. J. C 40, 121 (2005); M. Bordag and V. Skalozub, Eur. Phys. J. Plus 134, 289 (2019); O. A. Borisenko and V. V. Skalozub, arXiv:2002.05008.

[17] C. P. Korthals Altes, Nucl. Phys. B420, 637 (1994).

[18] P. Giovannangeli and C. P. Korthals Altes, Nucl. Phys. B721, 1 (2005); B721, 25 (2005).
[19] C. P. Korthals Altes (to be published).

[20] A. Dumitru, Y. Guo, Y. Hidaka, C. P. K. Altes, and R. D. Pisarski, Phys. Rev. D 83, 034022 (2011); A. Dumitru, Y. Guo, and C.P. Korthals Altes, Phys. Rev. D 89, 016009 (2014).

[21] Y. Guo, J. High Energy Phys. 11 (2014) 111.

[22] Y. Guo and Q. Du, J. High Energy Phys. 05 (2019) 042.

[23] C. P. K. Altes, R. D. Pisarski, and A. Sinkovics, Phys. Rev. D 61, 056007 (2000).

[24] C. P. Korthals Altes, H. Nishimura, R. D. Pisarski, and V. V. Skokov, Phys. Lett. B 803, 135336 (2020).

[25] C. P. Korthals-Altes and H. Nishimura (to be published).

[26] Y. Hidaka and R. D. Pisarski, Phys. Rev. D 80, 036004 (2009).

[27] A. Dumitru, Y. Guo, Y. Hidaka, C. P. K. Altes, and R. D. Pisarski, Phys. Rev. D 86, 105017 (2012).

[28] H. Nishimura and M. C. Ogilvie, Phys. Rev. D 85, 065021 (2012).

[29] A. Dumitru, J. Lenaghan, and R. D. Pisarski, Phys. Rev. D 71, 074004 (2005).

[30] R. D. Pisarski and V. V. Skokov, Phys. Rev. D 86, 081701 (2012).

[31] H. Nishimura, R. D. Pisarski, and V. V. Skokov, Phys. Rev. D 97, 036014 (2018).

[32] E. Brezin, C. Itzykson, G. Parisi, and J. Zuber, Commun. Math. Phys. 59, 35 (1978); D. Gross and E. Witten, Phys. Rev. D 21, 446 (1980); S. R. Wadia, Phys. Lett. 93B, 403 (1980).

[33] C. B. Lang, P. Salomonson, and B. S. Skagerstam, Nucl. Phys. B190, 337 (1981); P. Menotti and E. Onofri, Nucl. Phys. B190, 288 (1981); J. Jurkiewicz and K. Zalewski, Nucl. Phys. B220, 167 (1983); F. Green and F. Karsch, Nucl. Phys. B238, 297 (1984); P. H. Damgaard and A. Patkos, Phys. Lett. B 172, 369 (1986); S. I. Azakov, P. Salomonson, and B. S. Skagerstam, Phys. Rev. D 36, 2137 (1987); K. Demeterfi, N. Deo, S. Jain, and C.-I. Tan, Phys. Rev. D 42, 4105 (1990); J. Jurkiewicz, Phys. Lett. B 245, 178 (1990); B. Sundborg, Nucl. Phys. B573, 349 (2000); O. Aharony, J. Marsano, S. Minwalla, K. Papadodimas, and M. Van Raamsdonk, Adv. Theor. Math. Phys. 8, 603 (2004); Phys. Rev. D 71, 125018 (2005); L. Alvarez-Gaume, C. Gomez, H. Liu, and S. R. Wadia, Phys. Rev. D 71, 124023 (2005); H. J. Schnitzer, Nucl. Phys. B695, 267 (2004); T. J. Hollowood and J. C. Myers, J. High Energy Phys. 11 (2009) 008; S. Hands, T. J. Hollowood, and J. C. Myers, J. High 
Energy Phys. 07 (2010) 086; T. J. Hollowood, S. P. Kumar, and J. C. Myers, J. High Energy Phys. 11 (2011) 138; T. J. Hollowood and J. C. Myers, J. High Energy Phys. 10 (2012) 067; M. C. Ogilvie, J. Phys. A 45, 483001 (2012); Y. Liu, P. Warchol, and I. Zahed, Phys. Lett. B 753, 65 (2016).

[34] R. D. Pisarski, Phys. Rev. D 62, 111501 (2000); A. Dumitru and R. D. Pisarski, Phys. Lett. B 525, 95 (2002); Phys. Rev. D 66, 096003 (2002). O. Scavenius, A. Dumitru, and J. Lenaghan, Phys. Rev. C 66, 034903 (2002); A. Dumitru, Y. Hatta, J. Lenaghan, K. Orginos, and R. D. Pisarski, Phys. Rev. D 70, 034511 (2004); A. Dumitru, J. Lenaghan, and R. D. Pisarski, Phys. Rev. D 71, 074004 (2005); A. Dumitru, R. D. Pisarski, and D. Zschiesche, Phys. Rev. D 72, 065008 (2005); M. Oswald and R. D. Pisarski, Phys. Rev. D 74, 045029 (2006); R. D. Pisarski, Phys. Rev. D 74, 121703 (2006); A. Dumitru and D. Smith, Phys. Rev. D 77, 094022 (2008); D. Smith, A. Dumitru, R. Pisarski, and L. von Smekal, Phys. Rev. D 88, 054020 (2013).

[35] A. Dumitru, Y. Guo, Y. Hidaka, C. P. Korthals-Altes, and R. D. Pisarski, Phys. Rev. D 83, 034022 (2011); Phys. Rev. D 86, 105017 (2012).

[36] C. Sasaki and K. Redlich, Phys. Rev. D 86, 014007 (2012).

[37] R. D. Pisarski and V. V. Skokov, Phys. Rev. D 86, 081701 (2012); S. Lin, R. D. Pisarski, and V. V. Skokov, Phys. Rev. D 87, 105002 (2013).

[38] K. Kashiwa, R. D. Pisarski, and V. V. Skokov, Phys. Rev. D 85, 114029 (2012); K. Kashiwa and R. D. Pisarski,
Phys. Rev. D 87, 096009 (2013); C. Gale, Y. Hidaka, S. Jeon, S. Lin, J.-F. Paquet, R. D. Pisarski, D. Satow, V. Skokov, and G. Vujanoivic, Phys. Rev. Lett. 114, 072301 (2015); Y. Hidaka, S. Lin, R. D. Pisarski, and D. Satow, J. High Energy Phys. 10 (2015) 005; D. Satow and W. Weise, Phys. Rev. D 92, 056001 (2015); S. Lin, R. D. Pisarski, and V. V. Skokov, Phys. Lett. B 730, 236 (2014).

[39] R. D. Pisarski and V. V. Skokov, Phys. Rev. D 94, 034015 (2016); A. Folkestad and J. O. Andersen, Phys. Rev. D 99, 054006 (2019).

[40] K. Shiraishi and S. Hirenzaki, Z. Phys. C 53, 91 (1992); O. K. Kalashnikov, Phys. Lett. B 302, 453 (1993); S. Chapman, Phys. Rev. C 47, 1763 (1993).

[41] N. K. Nielsen, Nucl. Phys. B101, 173 (1975).

[42] H. Nishimura, C. Korthals-Altes, R. D. Pisarski, and V. Skokov, Proc. Sci. Confinement2018 (2019) 155 [arXiv: 1811.11736].

[43] A. Dumitru, Y. Hatta, J. Lenaghan, K. Orginos, and R. D. Pisarski, Phys. Rev. D 70, 034511 (2004).

[44] E. Poppitz and M. Unsal, J. High Energy Phys. 03 (2009) 027; M. Shifman and M. Unsal, Phys. Lett. B 681, 491 (2009); E. Poppitz, T. Schaefer, and M. Unsal, J. High Energy Phys. 10 (2012) 115; G. V. Dunne and M. Unsal, Annu. Rev. Nucl. Part. Sci. 66, 245 (2016); T. Kanazawa, M. Unsal, and N. Yamamoto, Phys. Rev. D 96, 034022 (2017).

[45] Y. Hidaka and R. D. Pisarski (to be published). 